\title{
Phase-averaged flow statistics in compressors using a rotated hot-wire technique
}

\author{
T. O. Jelly ${ }^{1}$ I. J. Day ${ }^{1}$ L. di Mare ${ }^{2}$
}

Received: 23 November 2016 / Revised: 25 February 2017 / Accepted: 27 February 2017 / Published online: 3 April 2017

(C) The Author(s) 2017. This article is an open access publication

\begin{abstract}
A technique based on a rotated hot wire has been developed to characterise the unsteady, three-dimensional flow field between compressor blade rows. Data are acquired from a slanted hot wire rotated through a number of orientations at each measurement point. Phase-averaged velocity statistics are obtained by solving a set of sensor response equations using a weighted, non-linear regression algorithm. The accuracy and robustness of the method were verified a priori by conducting a series of tests using synthetic data. The method is demonstrated by acquiring a full set of phase-averaged flow statistics in the wake of a compressor stator blade row. The technique allows three components of phase-averaged velocity, six components of phase-averaged deterministic stress, and six components of phase-averaged Reynolds stress to be recovered using a single rotated hot-wire probe.
\end{abstract}

Keywords Hot-wire anemometry · Turbulence . Turbomachinery

\section{Introduction}

The fluid motion that develops between compressor blade rows is exceptionally complex. The relative motion of alternate rotating and non-rotating blade rows (see Fig. 1)

T. O. Jelly

toj23@cam.ac.uk

1 Whittle Laboratory, Engineering Department, University of Cambridge, Cambridge, UK

2 Mechanical Engineering Department, Imperial College London, London, UK produces a flow field that is inherently unsteady, threedimensional and multi-scale.

It is customary to divide the unsteadiness in compressors into two categories: (1) deterministic fluctuations related to the shaft and blade frequencies and (2) broadband stochastic fluctuations related to naturally occurring turbulence. The mean square of (1) and (2) are manifest as deterministic stresses and Reynolds stresses, respectively. Furthermore, statistical moments may exhibit periodicities in both space and time. The space-time dependence of flow statistics significantly complicates the closure of the phase-averaged Navier-Stokes equations, relative to the traditional Reynolds-averaged formulation (Reynolds and Hussain 1972).

The numerical prediction of compressor performance and stability rank amongst the greatest achievements of modern computational fluid dynamics (CFD) (Bradshaw 1996). Several frameworks for compressor CFD exist and include: (1) the steady-state mixing-plane method (Denton 1990; Dawes 1991; (2) the unsteady sliding-plane method (Rai 1989); and (3) the average-passage method (Adamczyk 1985). Depending on which method is adopted, the action of deterministic stresses, Reynolds stresses, or their combined effect should be modeled properly to achieve physically meaningful results. However, it is widely acknowledged that contemporary modeling practice can compromise the reliability of CFD results (Denton 2010). Ultimately, modeling uncertainties will only be reduced through improved physical understanding.

To improve the understanding of momentum and energy exchange mechanisms in compressor annuli, it is imperative to acquire scale-resolved measurements in realistic, rotating, multi-bladed environments. Steady-state data are of limited value, since statistical moments of fluctuating quantities cannot be evaluated. Useful data must be highly 


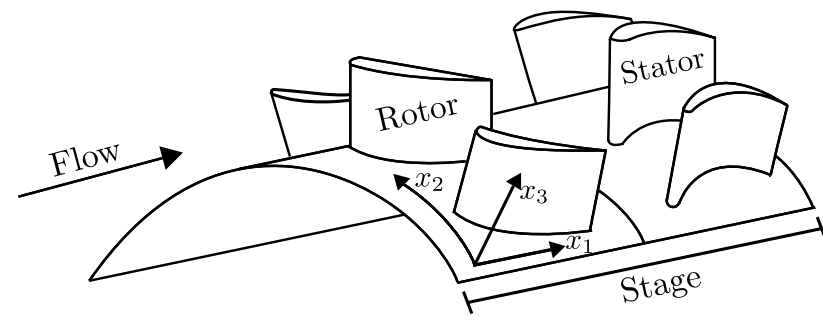

Fig. 1 Sketch of a compressor stage showing the axial $\left(x_{1}\right)$, circumferential $\left(x_{2}\right)$, and radial $\left(x_{3}\right)$ directions. Note that the outer casing of the compressor has been removed. This figure has been adapted from Bradshaw et al. (1981)

resolved in both space and time, so that statistics of the unsteady, three-component flow can be examined in detail. Therefore, the development of practical experimental techniques which return a complete set of phase-averaged flow statistics in realistic compressor environments is of significant interest.

In the past, a number of experimental methods have been used to characterise compressor flow fields. These methods include: triple-element hot-wire probes (Lakshminarayana and Poncet 1974), fast-response total-pressure probes (Zierke and Okiishi 1982), laser doppler anemometry (Faure et al. 2001), and particle image velocimetry (Uzol et al. 2002). Some of these methods are described in further detail by Lakshminarayana (1981) and Sieverding et al. (2000). This work aims to complement past methods by acquiring phase-locked flow statistics using a a technique based on a rotated hot wire.

\subsection{The rotated hot-wire technique}

The rotated hot-wire technique was first mentioned by Dryden (1938). He described a procedure to measure the Reynolds shear stress in a turbulent boundary layer based on the difference between two measurements acquired at different sensor orientations. Following work done by Prandtl (1946), Hinze (1959), and Webster (1962), theoretical models of the sensor's angular response were established and more sophisticated rotated hot-wire techniques were developed. These methods have been used to characterise the turbulence structure in flow configurations that include: square ducts (Brundrett and Baines 1964); circular pipes (Fujita and Kovasznay 1968; Papavegros and Hedley 1979); circumferentially strained boundary layers (Bissonnette and Mellor 1974); curved rectangular ducts (De Grande and Kool 1981); and downstream of turbulence-generating grids (Sirivat 1989). In addition, a number of rotated hot-wire methods have been developed and employed in compressors.

Schmidt and Okiishi (1977) developed a rotated slantwire method to measure periodic variations of velocity between the blade rows of a three-stage axial-flow compressor. A once-per-rotor-revolution trigger was used to phase-lock slanted hot-wire readings acquired at six different orientations. Following the solution of a set of nonlinear equations, the mean velocity vector was recovered. Kuroumaru et al. (1982) devised a similar method whereby phase-locked, slant-wire measurements were collected downstream of an impeller at 12 different probe orientations. Based on a least squares solution of the ensembleaveraged data, three components of mean flow and six components of Reynolds stress were recovered. Inoue and Kuromaru (1984) applied a similar technique to study the dynamics of vortical structures downstream of a rotating blade row. A 12-orientation method was also used by Goto (1991), who studied the mean-flow field and Reynolds stresses across a stator blade row in a low-speed, singlestage compressor. Shin et al. (1994) examined rotor-rotor interactions in a counter-rotating unducted fan by traversing a rotated slant-wire upstream, downstream, and in-between the rotating blade rows. Hsu and Wo (1997) measured the three-dimensional wake structure downstream of a compressor rotor blade row using a rotated slant wire to quantify rotor-stator interference effects. Ma and Jiang (2004) examined radial profiles of mean velocity, turbulence intensity, and Reynolds shear stresses, based on rotated slantwire measurements collected in the wake of a compressor rotor blade. In a recent study, Berdanier and Key (2016) devised a new data reduction technique for post-processing rotated slant-wire measurements. In this work, data were acquired in the tip region of a high-speed compressor at three different sensor orientations-effectively mimicking a triple-wire arrangement at each measurement point-and the time-mean pitch and yaw angles were recovered using a new look-up table method. Relative to the iterative solution of non-linear response equations, the look-up method required a fraction of the computational cost.

As an alternative to acquiring data with a rotated single wire, a multi-wire sensor could be used instead. Relative to a single wire, benefits of using a multi-wire sensor include: reduced total measurement time due to simultaneous wire sampling and direct evaluation of joint statistical properties in the amplitude, time, and frequency domains. Despite these advantages, multi-wire measurements in turbomachinery flows are known to suffer from the effects of prong interference (Díaz et al. 2016). As a result, only single-wire sensors were used throughout this work.

\subsection{Motivation and objectives}

Despite numerous measurements campaigns, details of phase-averaged deterministic stresses and phase-averaged Reynolds stresses between compressor blade rows remain scarce in the literature. To the best of the authors' 
knowledge, a rotated hot-wire method capable of resolving the time variation of deterministic and Reynolds stresses between compressor blade rows does not exist. The objective of this work is to develop such a method, with the aim of characterising the flow between compressor blade rows to a high level of statistical detail. This work establishes the theoretical, numerical, and experimental frameworks required to recover the phase-averaged velocity vector, phase-averaged deterministic stress tensor, and phase-averaged Reynolds stress tensor using a single rotated slant wire.

This paper is divided into six sections. Section 2 describes the derivation of the phase-averaged sensor response equations. Section 3 describes how the response equations are solved using a non-linear regression algorithm and the results from a series of a priori tests. Section 4 describes the experimental setup, including details of the compressor facility, data acquisition system, and calibration procedures. Section 5 details the experimental results, including phase-averaged velocities, deterministic velocities, phase-averaged deterministic stresses, and phase-averaged Reynolds stresses. Finally, in Sect. 6, the findings of this work are summarised.

\section{Phase-averaged sensor response equations}

This section describes the formulation of the phase-averaged sensor response equations and is divided into three parts. First, a relationship between the rotated, wire-fixed co-ordinate system, and the stationary compressor coordinate system is established. Second, a triple decomposition of the instantaneous velocity is invoked and equations for mean, deterministic, and stochastic components are derived. Finally, a set response equations which relate the flow statistics measured in the rotated, wire-fixed frame to the velocity statistics in the stationary compressor co-ordinate system are derived.

\subsection{Co-ordinate systems}

Throughout this work, the wire-fixed co-ordinate system of the slanted hot wire is designated $y_{i}$. With reference to Fig. 2, the $y_{1}$ axis lies tangential to the wire, the $y_{2}$ axis lies normal to the plane containing the sensor prongs, and the $y_{3}$ axis lies bi-normal to wire. The velocity components in the tangential $\left(y_{1}\right)$, normal $\left(y_{2}\right)$, and bi-normal $\left(y_{3}\right)$ directions are $v_{1}, v_{2}$, and $v_{3}$, respectively.

The purpose of this rotated hot-wire method is to recover a set of velocity statistics defined in the stationary compressor co-ordinate system, $x_{i}$, based on a number of measurements acquired in the wire-fixed frame, $y_{i}$. With reference to Fig. 3, the probe stem is aligned with the $x_{3}$ axis and the probe

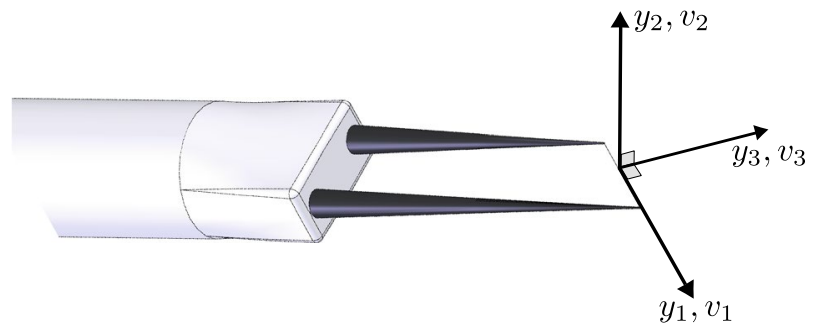

Fig. 2 Schematic diagram illustrating the wire-fixed co-ordinate system $y_{i}$. The velocity components in tangential $\left(y_{1}\right)$, normal $\left(y_{2}\right)$, and bi-normal $\left(y_{3}\right)$ directions are $v_{1}, v_{2}$, and $v_{3}$, respectively
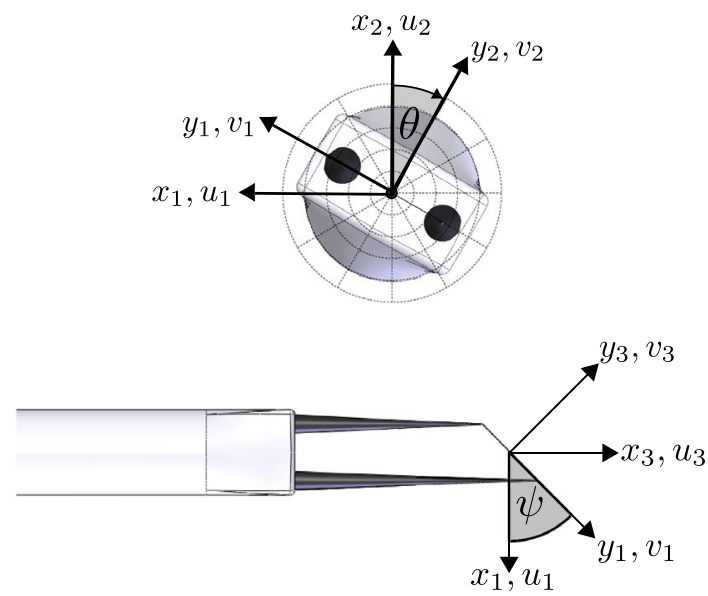

Fig. 3 Schematic diagrams illustrating the wire-fixed co-ordinate system, $y_{i}$, stationary co-ordinate system, $x_{i}$, probe setting angle, $\theta$, and wire slant angle, $\psi$

setting angle, $\theta$, is the angle formed between the rotated $y_{12}$ plane and stationary $x_{12}$ plane. Note that a positive change in $\theta$ corresponds to an anti-clockwise rotation around the probe stem axis, $x_{3}$. The probe setting angle is defined as zero when the $x_{1}$ axis, the sensor, and the prongs are coplanar and when the short prong lies upstream of the long prong. The wire slant angle, $\psi$, is the angle formed between the $y_{13}$ and $x_{13}$ planes and is held fixed at $45^{\circ}$.

A velocity vector $u_{i}$ in the compressor co-ordinate system and its representation $v_{i}$ in the wire-fixed co-ordinate system are related by the linear transformation

$v_{i}=M_{i j} u_{j}$

where

$M_{i j}=\left[\begin{array}{ccc}\cos \psi \cos \theta & \cos \psi \sin \theta & \sin \psi \\ -\sin \theta & \cos \theta & 0 \\ -\sin \psi \cos \theta & -\sin \psi \sin \theta & \cos \psi\end{array}\right]$.

For a fixed probe orientation, the squared cooling velocity, $q$, can be evaluated using Jørgensen's equation (1971):

$q=\delta_{1}^{2} v_{1}^{2}+\delta_{2}^{2} v_{2}^{2}+\delta_{3}^{2} v_{3}^{2}$ 
where $\delta_{1}, \delta_{2}$, and $\delta_{3}$ are the tangential, normal, and bi-normal wire sensitivity coefficients, respectively. The velocity transformation (1) can be inserted into Jørgensen's Eq. (3) to obtain

$q=G_{h k} u_{h} u_{k}$

where

$G_{h k} \equiv \delta_{1}^{2} M_{1 h} M_{1 k}+\delta_{2}^{2} M_{2 h} M_{2 k}+\delta_{3}^{2} M_{3 h} M_{3 k}$.

Equation (4) relates the instantaneous cooling velocity to products of the Cartesian velocity for a known probe orientation. Next, a triple decomposition of the instantaneous cooling velocity (4) will be invoked to obtain expressions for the time-averaged, deterministic, and stochastic components.

\subsection{Triple decomposition of instantaneous variables}

For hot-wire measurements between compressor blade rows, it is useful to invoke a triple decomposition of the instantaneous cooling velocity:

$$
\begin{aligned}
q & =\bar{q}(\theta, \mathbf{x})+\tilde{q}(\theta, \mathbf{x}, t)+q^{\prime}(\theta, \mathbf{x}, t) \\
& =\langle q\rangle(\theta, \mathbf{x}, t)+q^{\prime}(\theta, \mathbf{x}, t)
\end{aligned}
$$

where $\bar{q}$ is the time-averaged component, $\tilde{q}$ is the deterministic fluctuation, and $q^{\prime}$ is the true stochastic fluctuation. The phase-average $\langle q\rangle$ is obtained by evaluating

$\langle q\rangle=\lim _{N \rightarrow \infty} \frac{1}{N} \sum_{n=0}^{N} q(\theta, \mathbf{x}, t+n \tilde{\tau})$

where $\tilde{\tau}$ is the rotor passing period and $N$ is the number of rotor revolutions. The deterministic fluctuation can be recovered by phase-averaging the triple decomposition (5) and rearranging to obtain

$\tilde{q}=\langle q\rangle-\bar{q}$.

After inserting the triple decomposition (5) in to the instantaneous Eq. (4), and then applying the standard rules of phase-averaging outlined by Hussain and Reynolds (1970), the following equations are obtained:

$$
\begin{aligned}
\bar{q}= & G_{h k}\left(\bar{u}_{h} \bar{u}_{k}+\overline{\tilde{u}_{h} \tilde{u}_{k}}+\overline{u_{h}^{\prime} u_{k}^{\prime}}\right) \\
\tilde{q}= & G_{h k}\left(\bar{u}_{h} \tilde{u}_{k}+\bar{u}_{k} \tilde{u}_{h}+\tilde{u}_{h} \tilde{u}_{k}-\overline{\tilde{u}_{h} \tilde{u}_{k}}\right. \\
& \left.+\left\langle u_{h}^{\prime} u_{k}^{\prime}\right\rangle-\overline{u_{h}^{\prime} u_{k}^{\prime}}\right) \\
q^{\prime}= & G_{h k}\left(\left\langle u_{h}\right\rangle u_{k}^{\prime}+\left\langle u_{k}\right\rangle u_{h}^{\prime}+u_{h}^{\prime} u_{k}^{\prime}-\left\langle u_{h}^{\prime} u_{k}^{\prime}\right\rangle\right) .
\end{aligned}
$$

In Eq. (8), the phase-averaged deterministic stresses are

$\tilde{u}_{h} \tilde{u}_{k}=\left(\left\langle u_{h}\right\rangle-\bar{u}_{h}\right)\left(\left\langle u_{k}\right\rangle-\bar{u}_{k}\right)$

and the phased-averaged Reynolds stresses are $\left\langle u_{h}^{\prime} u_{k}^{\prime}\right\rangle=\left\langle\left(u_{h}-\left\langle u_{h}\right\rangle\right)\left(u_{k}-\left\langle u_{k}\right\rangle\right)\right\rangle$.

Next, expressions that relate statistics of phase-averaged cooling velocity to the deterministic stresses (10) and Reynolds stresses (11) will be derived.

\subsection{Phase-averaged sensor response equations}

An equation for the phase-averaged cooling velocity, $\langle q\rangle$, can be obtained by summing time-averaged equation (7) and the deterministic equation (8) to give

$\langle q\rangle=G_{h k}\left(\left\langle u_{h}\right\rangle\left\langle u_{k}\right\rangle+\left\langle u_{h}^{\prime} u_{k}^{\prime}\right\rangle\right)$.

To solve the phase-averaged equation (12), an additional equation that relates the phase-averaged Reynolds stresses (11) to statistics of the stochastic cooling velocity is required. A suitable expression can be derived by taking the outer product of Eq. (9) and then phase-averaging, to obtain

$$
\begin{aligned}
\langle Q\rangle= & A_{i j h k}\left[\left\langle u_{h}^{\prime} u_{i}^{\prime}\right\rangle\left\langle u_{k}\right\rangle\left\langle u_{j}\right\rangle+\left\langle u_{k}^{\prime} u_{i}^{\prime}\right\rangle\left\langle u_{h}\right\rangle\left\langle u_{j}\right\rangle\right. \\
& \left.+\left\langle u_{h}^{\prime} u_{j}^{\prime}\right\rangle\left\langle u_{k}\right\rangle\left\langle u_{i}\right\rangle+\left\langle u_{k}^{\prime} u_{j}^{\prime}\right\rangle\left\langle u_{h}\right\rangle\left\langle u_{i}\right\rangle+C\right]
\end{aligned}
$$

where

$\langle Q\rangle=\lim _{N \rightarrow \infty} \frac{1}{N} \sum_{n=0}^{N} q^{\prime}(t+n \tilde{\tau}, \theta) q^{\prime}(t+n \tilde{\tau}, \theta)$

and where $A_{i j h k}$ is a rank four tensor defined here as

$A_{i j h k} \equiv G_{i j} G_{h k}$.

The last term on the right-hand side of Eq. (13) contains the third- and fourth-order velocity correlations and the product of the phase-averaged Reynolds stress tensor

$$
\begin{aligned}
C=\left\langle u_{h}\right\rangle & \left\langle u_{k}^{\prime} u_{i}^{\prime} u_{j}^{\prime}\right\rangle+\left\langle u_{k}\right\rangle\left\langle u_{h}^{\prime} u_{i}^{\prime} u_{j}^{\prime}\right\rangle+\left\langle u_{i}\right\rangle\left\langle u_{h}^{\prime} u_{k}^{\prime} u_{j}^{\prime}\right\rangle \\
+ & \left\langle u_{j}\right\rangle\left\langle u_{i}^{\prime} u_{h}^{\prime} u_{k}^{\prime}\right\rangle+\left\langle u_{h}^{\prime} u_{k}^{\prime} u_{i}^{\prime} u_{j}^{\prime}\right\rangle-\left\langle u_{h}^{\prime} u_{k}^{\prime}\right\rangle\left\langle u_{i}^{\prime} u_{j}^{\prime}\right\rangle .
\end{aligned}
$$

To quantify the contribution of the term $C$ towards the cooling velocity, a perturbation analysis of Eq. (13) was conducted. In the limit of a low turbulence $(<10 \%)$ intensity flow, the instantaneous velocities can be written as

$u_{i}=\left\langle u_{i}\right\rangle+\epsilon u_{i}^{\prime} \quad \epsilon \rightarrow 0$

which, after some manipulation, can be used to derive the following set of sensor response equations:

$$
\begin{aligned}
\langle q\rangle=G_{h k} & \left\langle u_{h}\right\rangle\left\langle u_{k}\right\rangle+\mathcal{O}\left(\epsilon^{2}\right) \\
\langle Q\rangle=\epsilon^{2} & A_{i j h k}\left[\left\langle u_{h}^{\prime} u_{i}^{\prime}\right\rangle\left\langle u_{k}\right\rangle\left\langle u_{j}\right\rangle+\left\langle u_{k}^{\prime} u_{i}^{\prime}\right\rangle\left\langle u_{h}\right\rangle\left\langle u_{j}\right\rangle\right. \\
+ & \left.\left\langle u_{h}^{\prime} u_{j}^{\prime}\right\rangle\left\langle u_{k}\right\rangle\left\langle u_{i}\right\rangle+\left\langle u_{k}^{\prime} u_{j}^{\prime}\right\rangle\left\langle u_{h}\right\rangle\left\langle w_{i}\right\rangle\right] \\
+ & \mathcal{O}\left(\epsilon^{3}\right) .
\end{aligned}
$$


Relative to the leading order terms, the term $C$ is at least an order of magnitude smaller. As a result, the contribution of the term $C$ towards the phase-averaged cooling velocity is herein considered negligible.

\section{Solution of the sensor response equations}

This section describes the solution of the sensor response equations and is divided into three parts. First, the weighted non-linear regression algorithm employed to solve the phase-averaged response equations is described. Second, the accuracy of the non-linear regression model is demonstrated by conducting a series of a priori tests using synthetic data. Finally, the robustness of the non-linear regression output against small changes to the input parameters is verified by means of a sensitivity analysis.

\subsection{Weighted non-linear regression method}

If phase-averaged cooling velocities are available at several probe setting angles, then the sensor response equations (12) and (13) can be solved simultaneously using a weighted non-linear regression algorithm. For each probe setting angle, the residual between the modeled and measured data is

$r_{l}=\langle q\rangle\left(\theta_{l}\right)-f_{l}\left(\theta_{l}\right)$

$s_{l}=\langle Q\rangle\left(\theta_{l}\right)-g_{l}\left(\theta_{l}\right)$

where $f$ and $g$ correspond to the right-hand side of the phase-averaged equations (12) and (13), respectively. The sum-squared residual equations (18) and (19) define the cost function:

$\mathcal{L} \equiv \frac{1}{2}\left(r_{l} r_{l}+\alpha s_{l} s_{l}\right)$

where $\alpha$ is a weighting constant. The cost function $\mathcal{L}$ is minimised using the Newton-Raphson algorithm:

$\mathbf{z}_{n+1}=\mathbf{z}_{n}+\gamma \boldsymbol{H}_{n}^{-1} \mathcal{J}_{n}$

where subscript $n$ denotes the iteration index, $\gamma$ is a relaxation parameter, and $\mathbf{z}$ is the solution vector of phase-averaged velocity statistics:

$$
\begin{aligned}
z= & {\left[\left\langle u_{1}\right\rangle,\left\langle u_{2}\right\rangle,\left\langle u_{3}\right\rangle, \ldots\left\langle u_{1}^{\prime} u_{1}^{\prime}\right\rangle,\left\langle u_{1}^{\prime} u_{2}^{\prime}\right\rangle,\left\langle u_{1}^{\prime} u_{3}^{\prime}\right\rangle,\left\langle u_{2}^{\prime} u_{2}^{\prime}\right\rangle,\right.} \\
& \left.\left\langle u_{2}^{\prime} u_{3}^{\prime}\right\rangle,\left\langle u_{3}^{\prime} u_{3}^{\prime}\right\rangle\right]^{T} .
\end{aligned}
$$

The Jacobian $\mathcal{J}$ and the Hessian $\mathcal{H}$ are assembled at each Newton-Raphson update by evaluating the first- and second-order partial derivatives of the cost function (20) with respect to solution vector $z_{i}$ :

$\mathcal{J}=r_{l} \frac{\partial r_{l}}{\partial z_{i}}+\alpha s_{l} \frac{\partial s_{l}}{\partial z_{i}}$
$\boldsymbol{H}=\frac{\partial r_{l}}{\partial z_{i}} \frac{\partial r_{l}}{\partial z_{i}}+\frac{\partial r_{l}^{2}}{\partial z_{i} \partial z_{i}}+\alpha\left(\frac{\partial s_{l}}{\partial z_{i}} \frac{\partial s_{l}}{\partial z_{i}}+\frac{\partial^{2} s_{l}}{\partial z_{i} \partial z_{i}}\right)$.

The Newton-Raphson algorithm (21) is initialised using values of $\left\langle u_{i}\right\rangle$ and $\left\langle R_{i j}\right\rangle$ obtained by solving the sensor response equations (16) and (17) separately. Equation (16) is solved using non-linear regression via Newton-Raphson algorithm, and Eq. (17) is solved using linear regression via $\mathrm{QR}$ factorisation. This two-step procedure returns values of $\left\langle u_{h}\right\rangle$ and $\left\langle R_{h k}\right\rangle$ which form the initial guess for the Newton-Raphson algorithm (21). Using this initialisation procedure, the cost function (20) is minimised to machine precision within ten iterations. The solution vector, $z_{i}$, is obtained by solving the linear system of equations via LU decomposition.

Next, the accuracy of the numerical solution to the sensor response equations is examined by performing a series of a priori tests using synthetic velocity statistics. As demonstrated by Buresti and Di Cocco (1987), numerical tests are a convenient way to forecast the performance of rotated hotwire methods before time-intensive measurement campaigns.

\subsection{A priori tests}

Velocity signals of the form

$\left[\begin{array}{l}u_{1}(t) \\ u_{2}(t) \\ u_{3}(t)\end{array}\right]=\left[\begin{array}{l}\bar{u}_{1} \\ \bar{u}_{2} \\ \bar{u}_{3}\end{array}\right]+\left[\begin{array}{l}\tilde{u}_{1}(t) \\ \tilde{u}_{2}(t) \\ \tilde{u}_{3}(t)\end{array}\right]+\left[\begin{array}{l}u_{1}^{\prime}(t) \\ u_{2}^{\prime}(t) \\ u_{3}^{\prime}(t)\end{array}\right]$

were synthesised to have known statistical properties. The magnitude of the mean axial velocity component was held fixed at $\bar{u}_{1}=1.0$, whereas the ratios $\bar{u}_{2} / \bar{u}_{1}$ and $\bar{u}_{3} / \bar{u}_{1}$ were varied systematically. The deterministic fluctuations, $\tilde{u}_{i}$, were modeled as a weighted sum of three sinusoids:

$\tilde{u}_{i}(t)=A \sum_{m=0}^{2} \frac{\sin }{\sqrt{(m+1)}}\left[\frac{2(m+1) \pi}{\tilde{\tau}}\right]$

where $A$ denotes the amplitude. The stochastic velocity fluctuations, $u_{i}^{\prime}$, were synthesised by correlating the output of a pseudo-random number generator with a Gaussian kernel. Reynolds shear stresses were simulated by taking linear combinations of the correlated sequences. The turbulence intensity was quantified using

$\mathrm{Tu} \equiv \sqrt{\frac{1}{3} \overline{u_{i}^{\prime} u_{i}^{\prime}}} \times 100$.

A total of three test fields were synthesised-details are listed in Table 1.

For each test field, velocities were converted into a cooling velocity using Jørgensen's equation (4) which was then phase-averaged. The sequences generated for each probe setting angle are independent: this corresponds to 
Table 1 Statistical properties of the three synthetic velocity fields, including details of the time-averaged velocities, $\bar{u}_{i}$, time-averaged deterministic stresses, $\overline{\tilde{u}_{i} \tilde{u}_{j}}$, time-averaged Reynolds stresses, $\overline{u_{i}^{\prime} u_{j}^{\prime}}$, and turbulence intensity, Tu

\begin{tabular}{|c|c|c|c|c|c|c|c|c|c|}
\hline Case & $\frac{\bar{u}_{2}}{\bar{u}_{1}}$ & $\frac{\bar{u}_{3}}{\bar{u}_{1}}$ & $\begin{array}{l}\overline{\tilde{u}_{1} \tilde{u}_{1}}, \overline{u_{1}^{\prime} u_{1}^{\prime}} \\
\left(\times 10^{-3}\right)\end{array}$ & $\begin{array}{l}\overline{\tilde{u}_{1} \tilde{u}_{2}}, \overline{u_{1}^{\prime} u_{2}^{\prime}} \\
\left(\times 10^{-3}\right)\end{array}$ & $\begin{array}{l}\overline{\tilde{u}_{1} \tilde{u}_{3}}, \overline{u_{1}^{\prime} u_{3}^{\prime}} \\
\left(\times 10^{-3}\right)\end{array}$ & $\begin{array}{l}\overline{\tilde{u}_{2} \tilde{u}_{2}}, \overline{u_{2}^{\prime} u_{2}^{\prime}} \\
\left(\times 10^{-3}\right)\end{array}$ & $\begin{array}{l}\overline{\tilde{u}_{2} \tilde{u}_{3}}, \overline{u_{2}^{\prime} u_{3}^{\prime}} \\
\left(\times 10^{-3}\right)\end{array}$ & $\begin{array}{l}\overline{\tilde{u}_{3} \tilde{u}_{3}}, \overline{u_{3}^{\prime} u_{3}^{\prime}} \\
\left(\times 10^{-3}\right)\end{array}$ & $\mathrm{Tu}(\%)$ \\
\hline $\mathrm{A}$ & 0.1 & 0.2 & $1.58,3.96$ & $-0.54,-1.86$ & $0.32,0.17$ & $0.19,1.69$ & $-0.11,-0.05$ & $0.06,1.86$ & 5.00 \\
\hline B & 0.2 & 0.3 & $3.32,13.5$ & $-0.99,-5.20$ & $0.68,2.71$ & $0.29,5.83$ & $-0.20,-3.80$ & $0.13,10.3$ & 10.0 \\
\hline $\mathrm{C}$ & 0.3 & 0.4 & $5.72,31.9$ & $-1.66,-10.8$ & $1.23,3.80$ & $0.52,13.8$ & $-0.38,-8.14$ & $0.29,21.4$ & 15.0 \\
\hline
\end{tabular}

Table 2 Absolute percentage errors on the time-averaged velocities, $\bar{u}_{i}$, time-averaged deterministic stresses, $\overline{\tilde{u}}_{i} \tilde{u}_{j}$, and time-averaged Reynolds stresses, $\overline{u_{i}^{\prime} u_{j}^{\prime}}$, relative to the target data listed in Table 1

\begin{tabular}{|c|c|c|c|c|c|c|c|c|c|c|c|c|c|c|c|}
\hline Case & $\bar{u}_{1}$ & $\bar{u}_{2}$ & $\bar{u}_{3}$ & $\overline{\tilde{u}_{1} \tilde{u}_{1}}$ & $\overline{u_{1}^{\prime} u_{1}^{\prime}}$ & $\overline{\tilde{u}_{1} \tilde{u}_{2}}$ & $\overline{u_{1}^{\prime} u_{2}^{\prime}}$ & $\overline{\tilde{u}_{1} \tilde{u}_{3}}$ & $\overline{u_{1}^{\prime} u_{3}^{\prime}}$ & $\overline{\tilde{u}_{2} \tilde{u}_{2}}$ & $\overline{u_{2}^{\prime} u_{2}^{\prime}}$ & $\overline{\tilde{u}_{2} \tilde{u}_{3}}$ & $\overline{u_{2}^{\prime} u_{3}^{\prime}}$ & $\overline{\tilde{u}_{3} \tilde{u}_{3}}$ & $\overline{u_{3}^{\prime} u_{3}^{\prime}}$ \\
\hline A & 0.14 & 0.17 & 0.12 & 0.24 & 0.16 & 0.45 & 0.22 & 0.12 & 0.31 & 0.43 & 0.27 & 0.29 & 0.12 & 0.33 & 0.25 \\
\hline B & 0.18 & 0.20 & 0.18 & 0.13 & 0.12 & 0.21 & 0.28 & 0.17 & 0.13 & 0.18 & 0.23 & 0.28 & 0.21 & 0.31 & 0.33 \\
\hline $\mathrm{C}$ & 0.21 & 0.22 & 0.12 & 0.17 & 0.16 & 0.12 & 0.33 & 0.22 & 0.42 & 0.37 & 0.27 & 0.31 & 0.25 & 0.34 & 0.45 \\
\hline
\end{tabular}

the situation of consecutive measurements being taken at a fixed point in the flow and rotating the probe about its stem. Cooling velocities were synthesised at 15 probe setting angles distributed equally on the intervals $\theta \in[0,105]^{\circ}$ and $\theta \in[255,345]^{\circ}$ in $15^{\circ}$ increments.

The accuracy of the non-linear regression algorithm was verified by comparing the values of the synthesised cooling velocity against the numerical prediction for each probe setting angle. Synthesised values of the phase-averaged cooling velocity, $\langle q\rangle$, are compared against the numerical prediction for case $\mathrm{C}$ in Fig. 4 . The $l_{\infty}$ norm, based on the difference between the synthesised and predicted data, was computed to be $\mathcal{O}\left(10^{-2}\right)$.

The absolute percentage error on the time-mean statistics, relative to the target data listed in Table 1, was quantified as

Error $\equiv \frac{\left\|\overline{\mathbf{z}}-\overline{\mathbf{z}}_{\text {target }}\right\|}{\left\|\overline{\mathbf{z}}_{\text {target }}\right\|} \times 100$.

The absolute percentage error on each time-mean statistic was computed to be less than $1 \%$-details are provided in Table 2.

\subsection{Sensitivity analysis}

In practice, the accuracy of the rotated hot-wire measurements will be susceptible to probe imperfections, probe positioning errors, and drift in the calibration coefficients. Therefore, it is important to quantify how small changes in the model input parameters

$\lambda_{m}=\left\{\psi, \delta_{1}, \delta_{2}, \delta_{3}, \theta_{l}\right\}$



Fig. 4 Phase-averaged cooling velocity $\langle q\rangle$ including the synthesised open circle and the predicted values $(+)$ for the high turbulence intensity case $\mathrm{C}$ (see Table 1)

affect the output of the non-linear regression model. Mathematically, the change in the non-linear regression output with respect to the model parameter set $\lambda_{m}$ can be written as

$$
\begin{aligned}
\frac{\partial z_{i}}{\partial \lambda_{m}}= & -\mathcal{J}^{-1}\left(\frac{\partial r_{l}}{\partial \lambda_{m}} \frac{\partial r_{l}}{\partial z_{i}}+r_{l} \frac{\partial^{2} r_{l}}{\partial \lambda_{m} \partial z_{i}}\right. \\
& \left.+\alpha\left(\frac{\partial s_{l}}{\partial \lambda_{m}} \frac{\partial s_{l}}{\partial z_{i}}+s_{l} \frac{\partial s_{l}^{2}}{\partial \lambda_{m} \partial z_{i}}\right)\right) .
\end{aligned}
$$

After evaluating the total derivative of the left-hand side of equation (27), and then applying the Cauchy-Schwarz inequality, the sensitivity of the non-linear regression output can be written as

$\left|d z_{i}\right| \leq\left|\frac{\partial z_{i}}{\partial \psi}\right||d \psi|+\left|\frac{\partial z_{i}}{\partial \delta_{m}}\right|\left|d \delta_{m}\right|+\left|\frac{\partial z_{i}}{\partial \theta_{l}}\right|\left|d \theta_{l}\right|$

where $|d \psi|,\left|d \delta_{m}\right|$, and $\left|d \theta_{l}\right|$ are the levels of uncertainty in the wire slant angle, directional sensitivity coefficients, 
and probe setting angles, respectively. For the purposes of this sensitivity analysis, the levels of uncertainty were held fixed at 5\%. The non-linear regression output was found to be most sensitive to the wire sensitivity coefficient normal to the plane of the wire and the prongs, $\delta_{2}$, followed by $\delta_{3}$ , $\delta_{1}, \theta$ and $\psi$, as indicated by the relative magnitude of the corresponding terms on the right-hand side of Eq. (28). The sensitivity of each flow statistic to $\delta_{2}$ can be written as

$$
\begin{aligned}
& \left|\frac{\partial z_{i}}{\partial \delta_{2}}\right|=\underbrace{\left|\frac{\partial \overline{u_{1}}}{\partial \delta_{2}}\right|}_{S_{1}}+\underbrace{\left|\frac{\partial \overline{u_{2}}}{\partial \delta_{2}}\right|}_{S_{2}}+\underbrace{\left|\frac{\partial \overline{u_{3}}}{\partial \delta_{2}}\right|}_{S_{3}}+\underbrace{\left|\frac{\partial \overline{u_{1}^{\prime} u_{1}^{\prime}}}{\partial \delta_{2}}\right|}_{S_{4}}+\underbrace{\left|\frac{\partial \overline{u_{1}^{\prime} u_{2}^{\prime}}}{\partial \delta_{2}}\right|}_{S_{5}} \\
& +\underbrace{\left|\frac{\partial \overline{u_{1}^{\prime} u_{3}^{\prime}}}{\partial \delta_{2}}\right|}_{S_{6}}+\underbrace{\left|\frac{\partial \overline{u_{2}^{\prime} u_{2}^{\prime}}}{\partial \delta_{2}}\right|}_{S_{7}}+\underbrace{\left|\frac{\partial \overline{u_{2}^{\prime} u_{3}^{\prime}}}{\partial \delta_{2}}\right|}_{S_{8}}+\underbrace{\left|\frac{\partial \overline{u_{3}^{\prime} u_{3}^{\prime}}}{\partial \delta_{2}}\right|}_{S_{9}} .
\end{aligned}
$$

Each of the above sensitivities, $S_{i}$, are shown in Fig. 5 for the highest turbulence intensity case $\mathrm{C}$ (see Table 1). At most, the sensitivities are of unit order and this confirms that the output of the non-linear regression model is robust against small changes to the input parameters.

\section{Experimental setup}

This section describes the experimental set up and is divided into three parts. First, descriptions of the research compressor facility and traversing mechanism are provided. Second, the hot-wire anemometry apparatus, sampling parameters, and calibration procedures are described. Finally, some preliminary hot-wire measurements are discussed and a validation case against an independent fivehole pressure probe measurement is presented.

\subsection{Axial-flow compressor facility and traverse system}

Measurements were acquired in a single-stage, axial-flow, low-speed compressor-a facility that has been used in

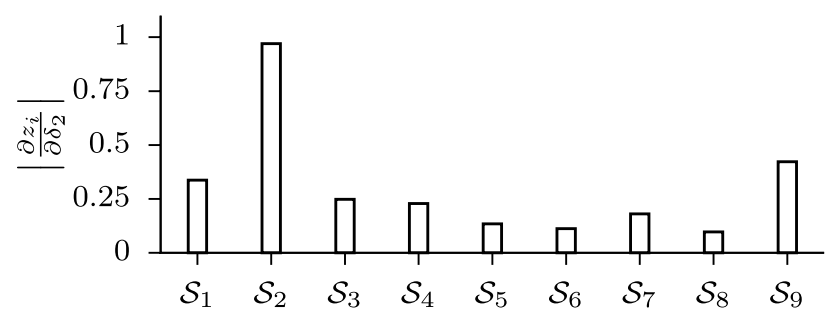

Fig. 5 Sensitivities of the non-linear regression output, $S_{i}$, to small changes in the normal directional coefficient, $\delta_{2}$, for the high turbulence intensity case $\mathrm{C}$
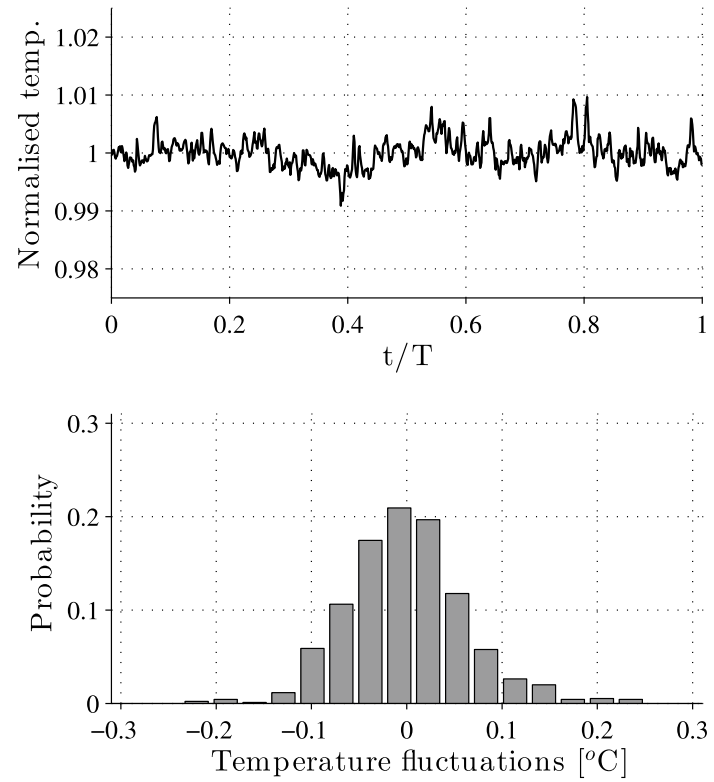

Fig. 6 Temperature variations measured at the rotor inlet including a time-history of normalised temperature and the probability density function of temperature fluctuations

previous studies (Goto 1991; Weichert and Day 2014). Ambient air is drawn into a $3400 \mathrm{~mm}$ diameter bell-mouth inlet through a thick gauze. The airstream passes through a honeycomb grid and two additional wire gauzes designed to straighten the flow. Following a 9:1 area contraction, the flow enters a parallel annulus with a mean radius of $762 \mathrm{~mm}$ and a hub-to-tip ratio of 0.8 . The airstream then passes through a turbulence grid, a row a 49 inlet guide vanes (IGV), 51 rotor blades, and 49 stator blades. The stator blades have a chord length of $132.4 \mathrm{~mm}$, a trailing edge diameter of $3 \mathrm{~mm}$, and a pitch-to-chord ratio of 0.8 . Downstream of the stator row trailing edge, the cylindrical annulus continues for an additional 20 stator chord lengths before the airstream exits into a dump diffuser. A conical throttle valve and an auxiliary fan positioned at the rear of the machine are used to set the flow rate through the compressor. The compressor was operated close to its design point, and the Reynolds number, based on the rotor speed at mid-height, $U_{m}$, and the stator chord, $c$, was held fixed at $2.4 \times 10^{5}$.

Before acquiring data, the temperature inside the compressor was allowed to settle into a statistically stationary state. By monitoring the output of a thermocouple positioned upstream of the rotor, a steady-state temperature was achieved after a settling period of around $30 \mathrm{~min}$. After this, the time-averaged rotor inlet temperature was acquired simultaneously during each hot-wire measurement. A time history of the rotor inlet temperature and the probability density function (PDF) of the fluctuations are shown in Fig. 6. For all the results included in this paper, 
the temperature fluctuations were within $\pm 0.25{ }^{\circ} \mathrm{C}$ of the mean value.

A three-axis traverse gear secured to the outer casing of the compressor was used to control the position and orientation of the slanted hot-wire sensor. A circumferential slot in the casing permitted traverses on the plane perpendicular to the compressor axis to be performed. A pair of stepper motors were used to control the radial and tangential positions of the probe. The probe setting angle was also controlled using a stepper motor with an angular resolution of $\Delta \theta= \pm 0.9^{\circ}$. The current traverse mechanism permits fully automated, rotated hot-wire measurements to be acquired from hub-to-casing across four stator passages. All rotated hot-wire measurements were acquired in the compressor stationary co-ordinate system, $x_{i}$.

The blade-to-blade plane of the compressor stage is shown in Fig. 7. The velocity components in the axial $\left(x_{1}\right)$, circumferential $\left(x_{2}\right)$, and radial $\left(x_{3}\right)$ directions are denoted $u_{1}, u_{2}$, and $u_{3}$, respectively. Measurements were acquired across two stator pitches at mid-span, at an axial distance $x_{1} / c=0.2$ from the stator trailing edge.

\subsection{Hot-wire anemometry and calibration procedure}

Hot-wire measurements were acquired using a constant temperature anemometer (CTA). The bridge voltage was digitised using a 16-bit analogue-to-digital-converter interfaced to the output of a BNC multiplexer. The sensor was operated with an overheat ratio of 1.8. The ratio of the sensor sampling frequency, $f_{s}$, to the shaft frequency was held fixed at $1.2 \times 10^{4}$. The sensor cut-off frequency, $f_{\mathrm{c}}$, was tuned to be half the value of $f_{\mathrm{s}}$. The slanted hot-wire probe has two straight prongs which support a $3 \mathrm{~mm}$ platinumplated tungsten wire inclined at a nominal angle of $45^{\circ}$, relative to the probe stem axis. The sensing element is etched halfway between the prongs to have a length-to-diameter ratio of 250 .

Before and after each measurement, the hot-wire sensor was calibrated against a Pitot-static probe housed in a stand-alone, round jet facility. A detailed description of the calibration tunnel is provided by di Mare et al. (2017). Hotwire measurements were acquired within the potential core of the jet, one nozzle diameter downstream of the nozzle exit, with the flow normal to the plane of the prongs. The squared sensor voltage, $E^{2}$, was correlated to the effective cooling velocity using the power law proposed by King (1914)

$E^{2}-E_{0}^{2}=B q^{\frac{n}{2}}$

where $E_{0}$ is the voltage measured at zero velocity and where $B$ and $n$ are the King's Law coefficients. By recording the bridge output across a range of known jet velocities,

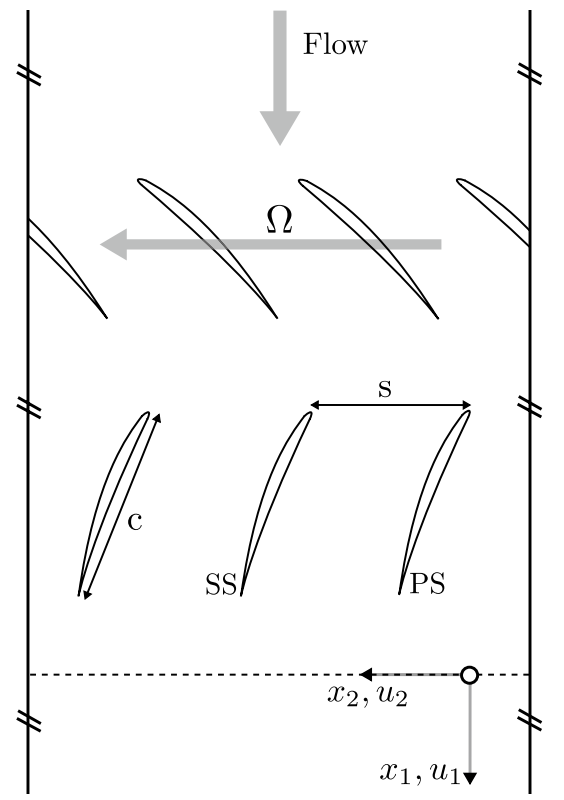

Fig. 7 Blade-to-blade plane at mid-span indicating the stator pitch, $s$, the stator chord, $c$, the suction-surface (SS), and the pressure-surface (PS) sides of the stator wake. All measurements were acquired across two stator pitches at an axial distance $x_{1} / c=0.2$ downstream from the stator row trailing edge (dashed lines)

the King's Law coefficients $B$ and $n$ were obtained using simple linear regression.

Throughout velocity calibration, the bridge voltage varied with both the velocity and temperature of the jet. Data from multiple velocity calibrations were collapsed on to a single master curve using a temperature correction of the form:

$E^{*}=\left(E-E_{0}\right)+\alpha \Delta T$

where $E^{*}$ denotes the temperature-corrected bridge voltage, $\alpha$ is a user-prescribed coefficient, and $\Delta T$ denotes the temperature difference, relative to a reference value. Four sets of temperature-corrected data are shown in Fig. 8 which collapse on to a master calibration curve using a value of $\alpha=0.008$ for temperature changes within the range $\Delta T \pm 2^{\circ} \mathrm{C}$.

The wire sensitivity coefficients, $\delta_{i}$, were determined via directional calibration. First, data were acquired at three fixed probe orientations, as shown in Fig. 9. The cooling velocity at each orientation was determined using the bridge voltage and King's Law coefficients. Using this data, the wire sensitivity coefficients were evaluated using the relation:

$\delta_{i}^{2}=\frac{\bar{q}_{\delta_{i}}}{\bar{q}_{\delta_{2}}}$ 


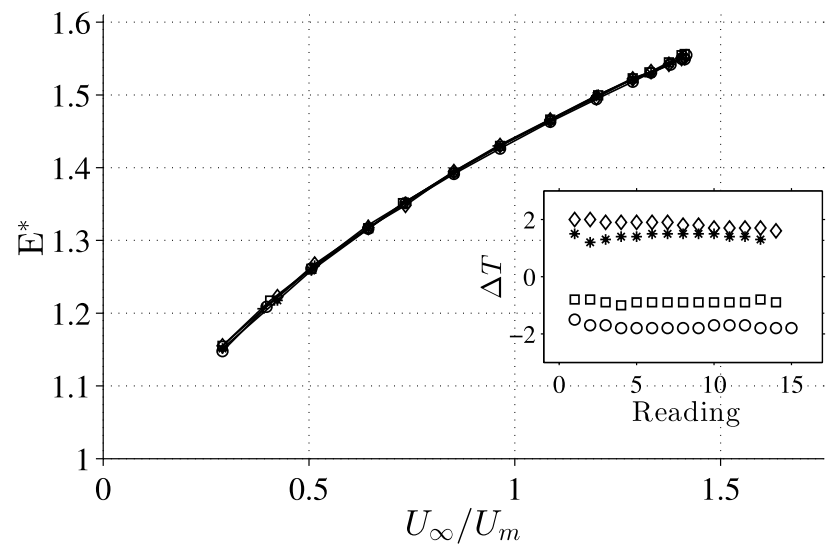

Fig. 8 Master calibration curve showing temperature-corrected voltages. The inset figure shows the temperature difference throughout velocity calibration. Note that the data have been normalised using the rotor speed at mid-height, $U_{m}$

where $\bar{q}_{\delta_{1}}, \bar{q}_{\delta_{2}}, \bar{q}_{\delta_{3}}$ denote the cooling velocity measured at tangential, normal, and bi-normal orientations, as shown in Fig. 9, respectively. Typical values of $\delta_{1}, \delta_{2}$ and $\delta_{3}$ were found to be $0.1,1.0$, and 0.9 , respectively. In addition to using Eq. (32), the values of $\delta_{i}$ were also estimated using a non-linear regression procedure. By acquiring data at different-but known-orientations of the wire and flow velocities in the calibration tunnel, the set of wire parameters

$\mathbf{z}=\left[\psi, \delta_{1}^{2}, \delta_{3}^{2}\right]^{\top}$

was determined by minimising the residual function

$r_{i}=E_{0}^{2}+B \bar{q}^{-\frac{n}{2}}\left(\mathbf{z}, \mathbf{u}_{i}, \theta_{i}\right)-E_{i}^{2}$

using the Newton-Raphson algorithm described in Sect. 3.1. The values of $\delta_{i}$ estimated via non-linear regression were within $5 \%$ of the values predicted using Eq. (32).
In addition, the wire slant angle $\psi$ was predicted to within $\pm 0.2^{\circ}$ of the measured value based on a magnified image of the sensor. With reference to the sensitivity analysis of the sensor response equations presented in Sect. 3.3, an uncertainty of $\left|d \delta_{i}\right| \approx 0.05$ will not significantly the alter the output of the non-linear regression algorithm. As a result, the values of $\delta_{1}, \delta_{2}$ and $\delta_{3}$ used throughout this work were $0.1,1.0$, and 0.9 , respectively.

The time-mean cooling velocity, $\bar{q}$, is compared against the velocity measured using the Pitot-static tube at 36 uniformly spaced probe setting angles (with the plane of the prongs perpendicular to the jet axis) in Fig. 10. The data sets match to within $3 \%$ for all probe setting angles outside the range $\theta \in(105,255)^{\circ}$. Within this range, the sensing element becomes immersed in the wake of the long prong, and as a result, significant levels of disagreement are observed.

\subsection{Statistical convergence and preliminary checks}

All hot-wire measurements acquired inside the compressor were recorded relative to a once-per-revolution rotor trigger, which was also used to determine the blade passing frequency:

$\beta=K \Omega$

where $K$ and $\Omega$ denote the number of rotor blades and rotor frequency, respectively. Throughout the measurement campaign, the rotor frequency had a nominal value of 528 revolutions per minute. Small deviations in the rotor frequency were accounted for by linearly interpolating each once-perrevolution record to have 6528 points. The interpolated data were ensemble-averaged over $N$ rotor revolutions to obtain phase-averaged statistics.

The convergence of time-mean statistics was verified using a hot-wire signal acquired at a fixed probe setting angle $\left(\theta=90^{\circ}\right)$. The effect of increasing the number of the rotor (a)

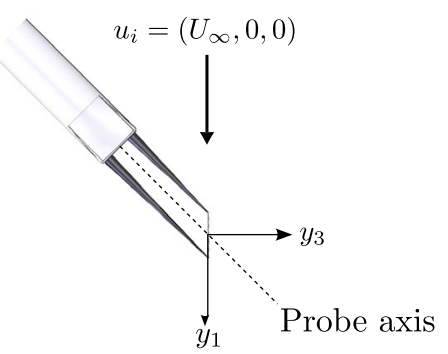

(b)

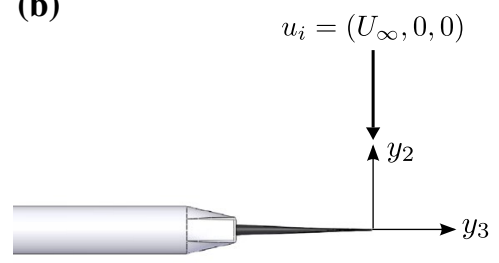

(c)

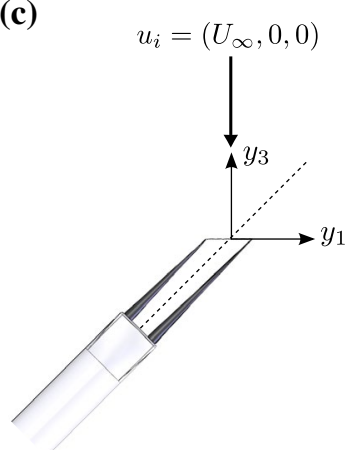

Fig. 9 Schematic diagram showing the orientation of the calibration jet velocity vector, $u_{i}=\left(U_{\infty}, 0,0\right)$, and the slanted hot-wire used to evaluate the a tangential $\left(\delta_{1}\right)$; $\mathbf{b}$ normal $\left(\delta_{2}\right)$ and $\mathbf{c}$ bi-normal $\left(\delta_{3}\right)$ wire sensitivity coefficients 


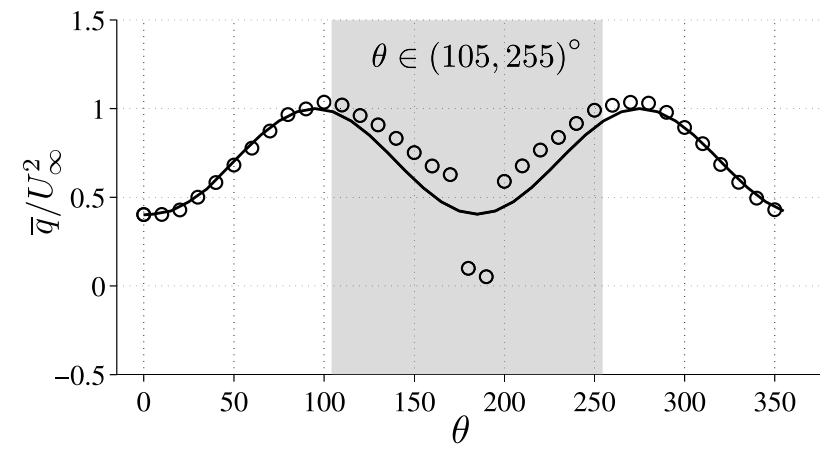

Fig. 10 Validation of sensor calibration procedure showing measured (open circle) and modeled (solid lines) mean cooling velocity, $\bar{q}$, at 36 uniformly spaced probe setting angles. Note that the data have been normalised using the jet velocity, $U_{\infty}$

revolutions, $N$, upon the mean-squared deterministic cooling velocity, $\overline{\tilde{q}^{2}}$, and the mean-squared stochastic cooling velocity, $\bar{q}^{2}$, at three tangential positions is shown in Fig. 11. Adequate convergence is achieved within a sampling period of 64 rotor revolutions at each tangential position.

A four-passage area traverse was conducted to verify circumferential periodicity around the annulus. Data were acquired at a fixed probe orientation $\left(\theta=90^{\circ}\right)$ for a sampling period of 64 rotor revolutions across an equi-spaced grid of 16 radial points and 96 tangential points. Contours of the mean cooling velocity, $\bar{q}$, are shown in Fig. 12-excellent levels of passage-to-passage periodicity are observed.

To identify the probe orientations susceptible to prong interference, a single-passage line traverse at mid-span was conducted. For each measurement point, the probe was rotated through one revolution in 24 equal increments. Interference was detected in the range $\theta \in[120,240]^{\circ}$ and these angles were omitted from all future measurements. After this, data were acquired at 15 different probe setting angles distributed equally on the intervals $\theta \in[0,105]^{\circ}$ and $\theta \in[255,345]^{\circ}$ in $15^{\circ}$ degree increments for each measurement point. For each probe orientation, the phase-averaged cooling velocities were computed using an ensemble of 64 phase-locked time series.

The accuracy and reliability of the rotated hot-wire results were validated against an independent five-hole pressure probe (5HP) measurement. The 5HP was calibrated using the non-nulling procedure described by Treaster and Yocum (1978). The time-averaged velocity vector, $\bar{u}_{i}$, based on the 5HP and rotated hot-wire measurements is compared in Fig. 13, which also includes a $95 \%$ confidence interval on the hot-wire data. After using the time-averaged velocity vector, $\bar{u}_{i}$, to evaluate the total velocity

$\left|u_{i}\right|=\sqrt{\bar{u}_{1}^{2}+\bar{u}_{2}^{2}+\bar{u}_{3}^{2}}$
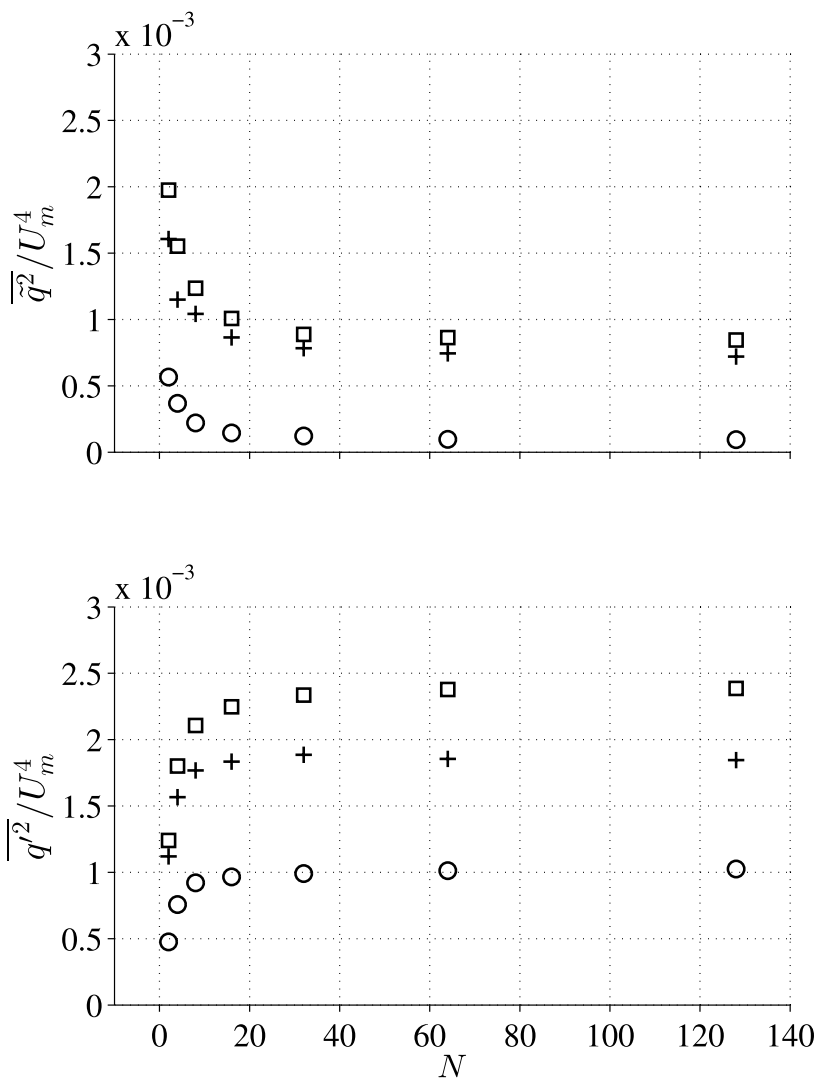

Fig. 11 Convergence of the mean-squared deterministic cooling velocity, $\overline{\tilde{q}^{2}}$, and mean-squared stochastic cooling velocity, $\overline{q^{\prime 2}}$, with increasing number of rotor revolutions, $N$. Data were acquired on the wake SS (open square), wake centre line (open circle), and wake PS $(+)$ and have been normalised using the rotor speed at mid-height, $U_{m}$

the maximum relative error on $\left|u_{i}\right|$ between the 5HP and hot-wire data was computed to be $9 \%$ which occurred on the wake suction side. An improved level of agreement may be possible by employing smaller, fast-response pressure probes. The steps required to fabricate, calibrate, and use miniaturised 5HP in turbomachinery flows have been discussed in detail by Georgiou and Milidonis (2014) and Grimshaw and Taylor (2016).

\section{Phase-averaged velocity statistics}

This section presents the phase-averaged velocity statistics downstream of the compressor stator and is divided into three parts. First, the phase-averaged and deterministic velocity are presented. Second, the six components of the phase-averaged deterministic stress tensor are presented. Finally, the six components of the phase-averaged Reynolds stress tensor are examined and compared against their deterministic counterpart. 
Fig. 12 Four-passage area traverse showing contours of mean cooling velocity, $\bar{q}$. The dashed line indicates the position of a two-passage traverse at mid-span. Data were acquired at a fixed probe orientation $\left(\theta=90^{\circ}\right)$ and have been normalised using the blade speed at mid-height, $U_{m}$

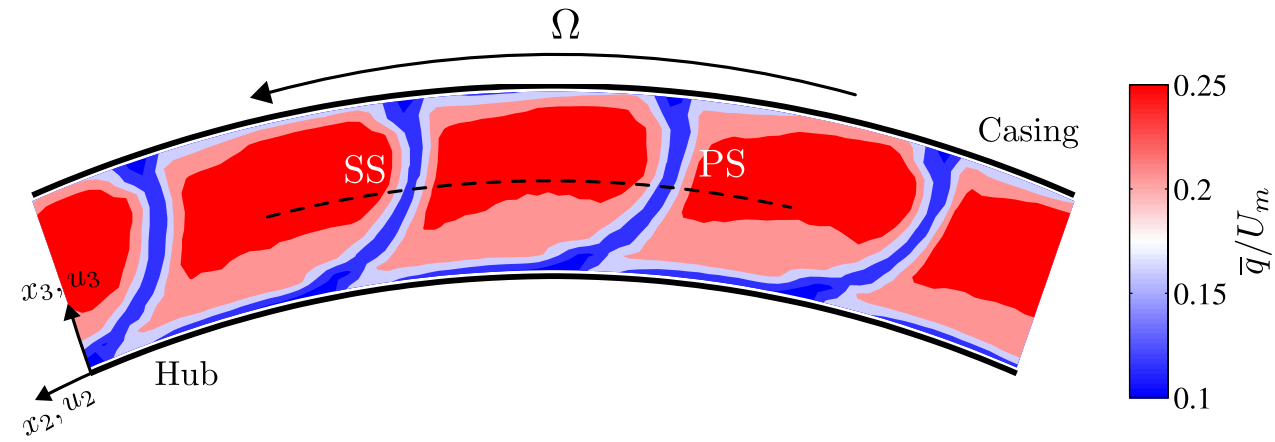

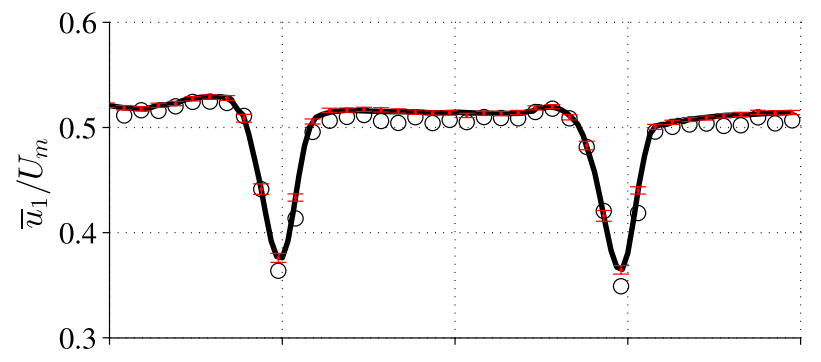
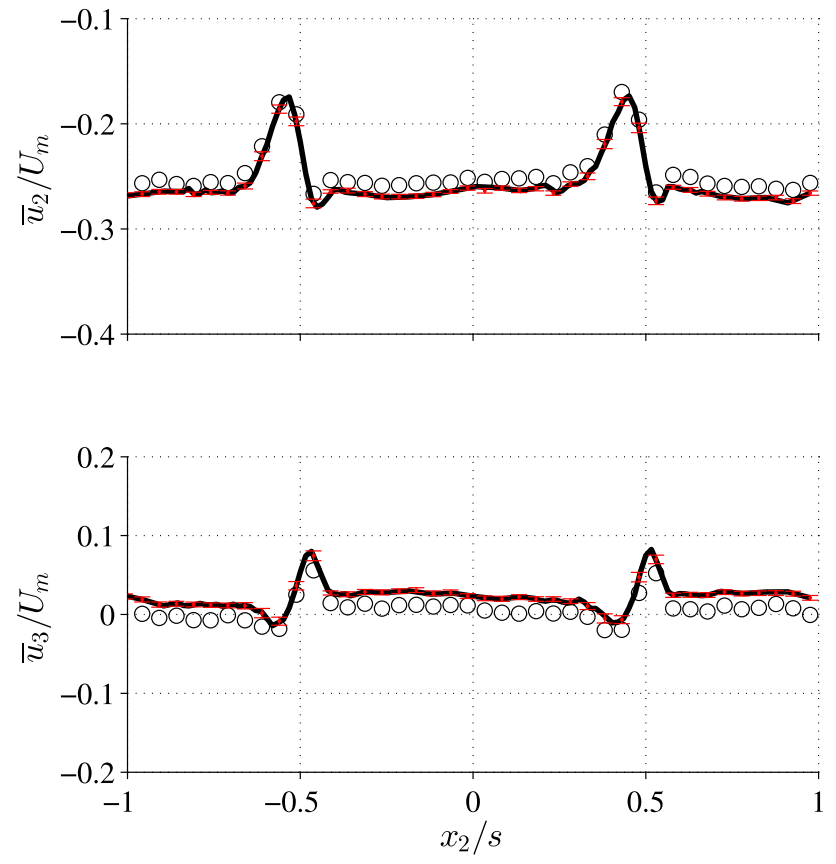

Fig. 13 Comparison of time-averaged velocity vector, $\bar{u}_{i}$, based on the rotated hot-wire (solid line) and 5HP (open square) measurements. A 95\% confidence interval on the hot-wire data (solid line) is also shown. Note that the data have been normalised using rotor speed at mid-height, $U_{m}$, and the stator pitch, $s$

\subsection{Phase-averaged and deterministic velocities}

The phase-averaged flow field downstream of the compressor stator is shown in Fig. 14. Space-time contours of the axial, $\left\langle u_{1}\right\rangle$, tangential, $\left\langle u_{2}\right\rangle$, and radial, $\left\langle u_{3}\right\rangle$, phase-averaged velocities are plotted for three blade passing periods. On either side of the stator wake centre line $\left(x_{2} / s \approx \pm 0.5\right)$, the passing rotor wakes are visible in the "free-stream" region $\left(-0.4<x_{2} / s<+0.4\right)$. The rotor wakes interact with the edges of the stator wake and cause the stator wake to meander in both space and time. The rotor-stator interactions can be clarified by evaluating the deterministic velocity:

$\tilde{u}_{i}\left(x_{2}, t\right)=\left\langle u_{i}\right\rangle\left(x_{2}, t\right)-\bar{u}_{i}\left(x_{2}\right)$.

The deterministic flow field downstream of the compressor stator is shown in Fig. 15. Space-time contours of the axial, $\tilde{u}_{1}$, tangential, $\tilde{u}_{2}$, and radial, $\tilde{u}_{3}$, deterministic velocities are plotted for three blade passing periods. Regions of negative deterministic velocity $\left(\tilde{u}_{i}<0\right)$ correspond to individual rotor wakes, which intersect each stator wake and tangentially coalesce across the passage. The interaction of the rotor and stator wakes leads to vigorous deterministic fluctuations within the stator wake, where the axial, tangential, and radial components achieve a maximum absolute value of $0.08,0.08$, and $0.04 U_{m}$, respectively. In general, the deterministic flow patterns shown in Fig. 15 are in-line with observations of Uzol et al. (2002), who interpreted compressor flow fields as a "lattice" of intertwined blade wakes.

\subsection{Phase-averaged deterministic stresses}

In the preceding subsection, details of the phase-averaged velocity (see Fig. 14) and deterministic velocity (see Fig. 15) downstream of the compressor stator were provided. In this subsection, the six components of the phaseaveraged deterministic stress tensor are presented and discussed.

The phase-averaged deterministic normal stresses downstream of the compressor stator are shown in Fig. 16. Space-time contours of the axial-axial, $\tilde{u}_{1} \tilde{u}_{1}$, tangential-tangential, $\tilde{u}_{2} \tilde{u}_{2}$, and radial-radial, $\tilde{u}_{3} \tilde{u}_{3}$, components are plotted for three blade passing periods. In the freestream region, the levels of deterministic normal stress can become very small $\left(\approx 0.01 U_{m}\right)$. On the other hand, considerable levels of time-varying deterministic normal stress 

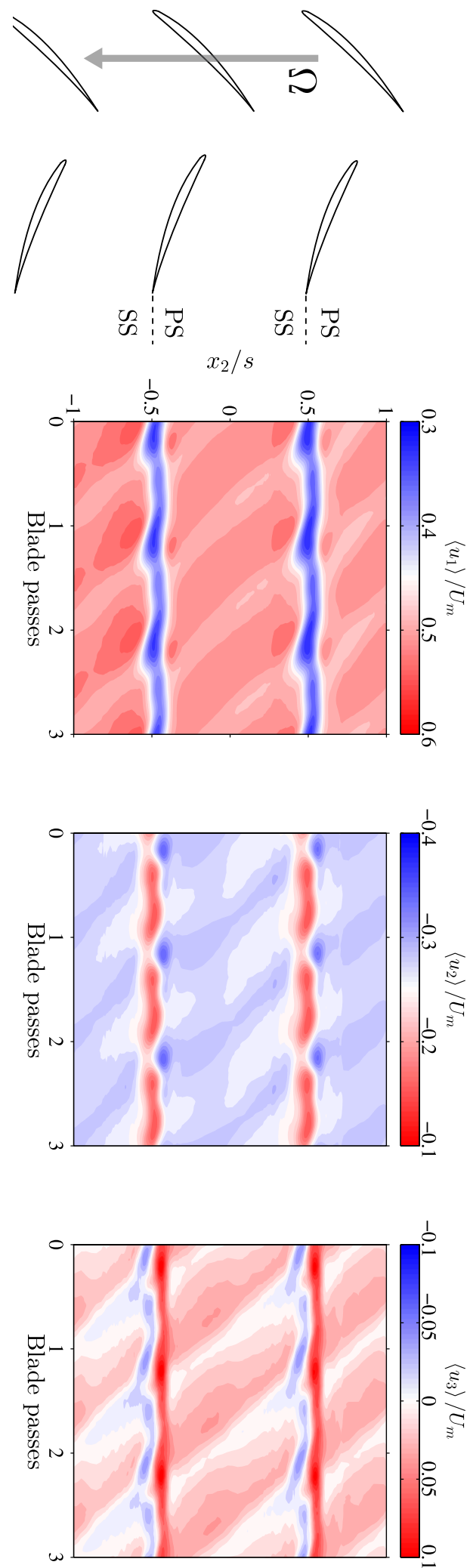

Fig. 14 Phase-averaged velocities including the axial $\left\langle u_{1}\right\rangle$, tangential $\left\langle u_{2}\right\rangle$, and radial $\left\langle u_{3}\right\rangle$ components. Note that data are shown for three blade passing periods and have been normalised using the rotor speed at mid-height, $U_{m}$, and the stator pitch, $s$
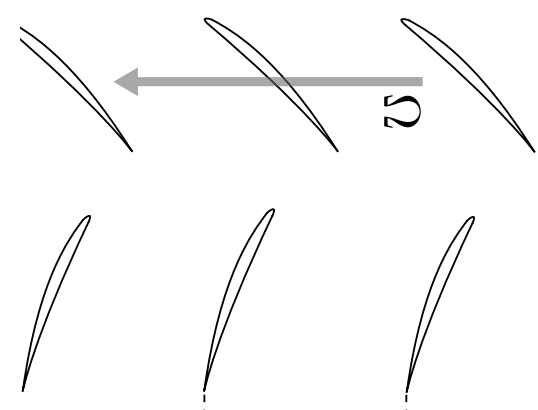

w

议:
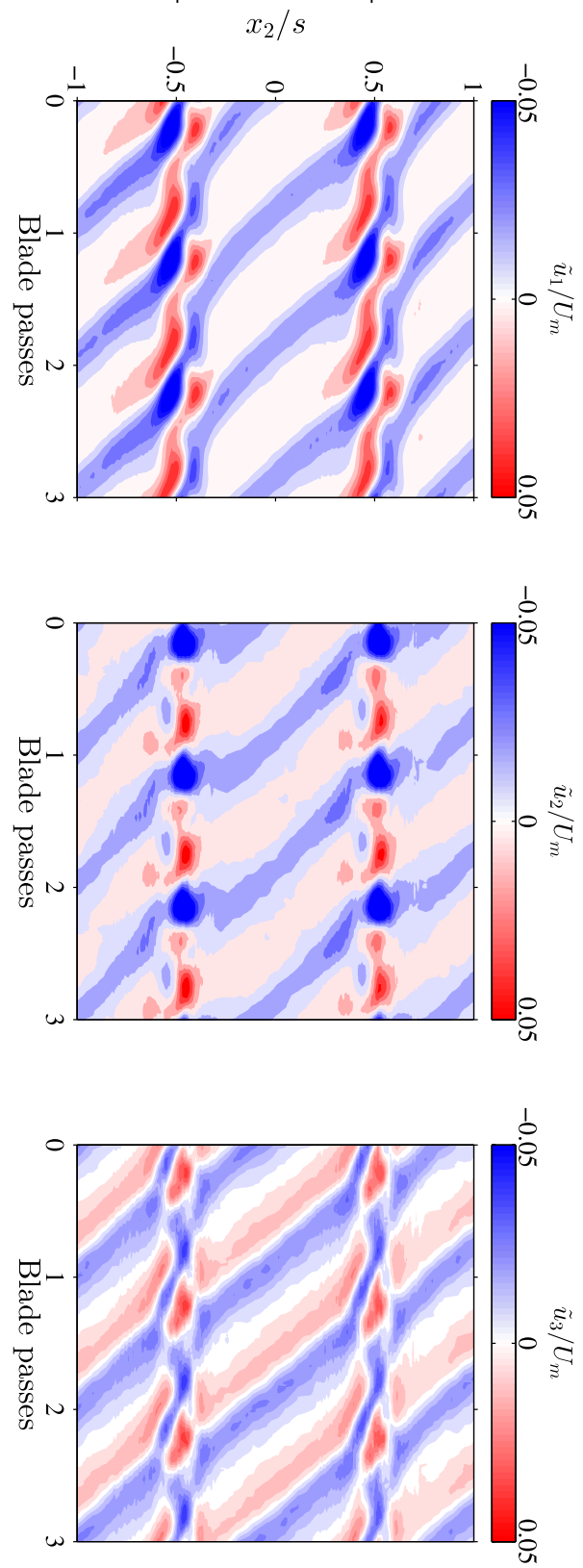

Fig. 15 Deterministic velocities including the axial $\tilde{u}_{1}$, tangential $\tilde{u}_{2}$, and radial $\tilde{u}_{3}$ components. Note that data are shown for three blade passing periods and have been normalised using the rotor speed at mid-height, $U_{m}$, and the stator pitch, $s$ 
occur within the stator wake-which agrees well with the deterministic velocity fields, as shown in Fig. 15. The time dependence of the normal stresses is related to the duty cycle of each rotor wake which supply a source of timeperiodic shear. In addition to their intermittency, the components of normal stresses are distributed asymmetrically about the stator wake centre line. The axial-axial and tangential-tangential stresses show a biased towards the stator wake SS and PS, respectively. The maximum and minimum values of the deterministic turbulence intensity

$\tilde{I}=\frac{1}{U_{m}} \sqrt{\frac{1}{3} \overline{\tilde{u}_{i} \tilde{u}_{i}}} \times 100$

were computed to be 3 and $1 \%$ and occur within the stator wake and free-stream regions, respectively. The circumferential average of deterministic turbulence intensity at midspan was computed to be $1.5 \%$.

The phase-averaged deterministic shear stresses downstream of the compressor stator is shown in Fig. 17. Space-time contours of the axial-tangential, $\tilde{u}_{1} \tilde{u}_{2}$, tangential-radial, $\tilde{u}_{2} \tilde{u}_{3}$, and axial-radial, $\tilde{u}_{1} \tilde{u}_{3}$, components are plotted for three blade passing periods. The distribution of deterministic shear stresses is qualitatively similar to the normal stresses, as shown in Fig. 17. The strongest shear stresses are produced intermittently within the stator wake. In addition, each component of deterministic shear-stress exhibits a characteristic sign change about the stator wake centre line. In terms of magnitude, the dominant deterministic shear stress is the axial-tangential component, $\tilde{u}_{1} \tilde{u}_{2}$, followed by the axial-radial component, $\tilde{u}_{1} \tilde{u}_{3}$, and, finally, the tangential-radial component, $\tilde{u}_{2} \tilde{u}_{3}$.

\subsection{Phase-averaged Reynolds stresses}

In the preceding subsection, details of the phase-averaged deterministic normal stresses (see Fig. 16) and the phaseaveraged deterministic shear stresses (see Fig. 17) were provided. In this subsection, the six components of the phase-averaged Reynolds stress tensor are presented and compared against their deterministic counterpart.

The phase-averaged Reynolds normal stresses downstream of the compressor stator are shown in Fig. 18. Space-time contours of the axial-axial, $\left\langle u_{1}^{\prime} u_{1}^{\prime}\right\rangle$, tangential-tangential, $\left\langle u_{2}^{\prime} u_{2}^{\prime}\right\rangle$, and radial-radial, $\left\langle u_{3}^{\prime} u_{3}^{\prime}\right\rangle$, components are plotted for three blade passing periods. Relative to the deterministic normal stresses shown previously in Fig. 16, the Reynolds normal stresses exhibit some striking differences. First, the Reynolds stresses within the stator wake show a significantly lower level of intermittency and are more "fully-developed" in character. Second, the Reynolds normal stress exhibits a dual-peaked distribution about the stator wake centre line-a defining feature
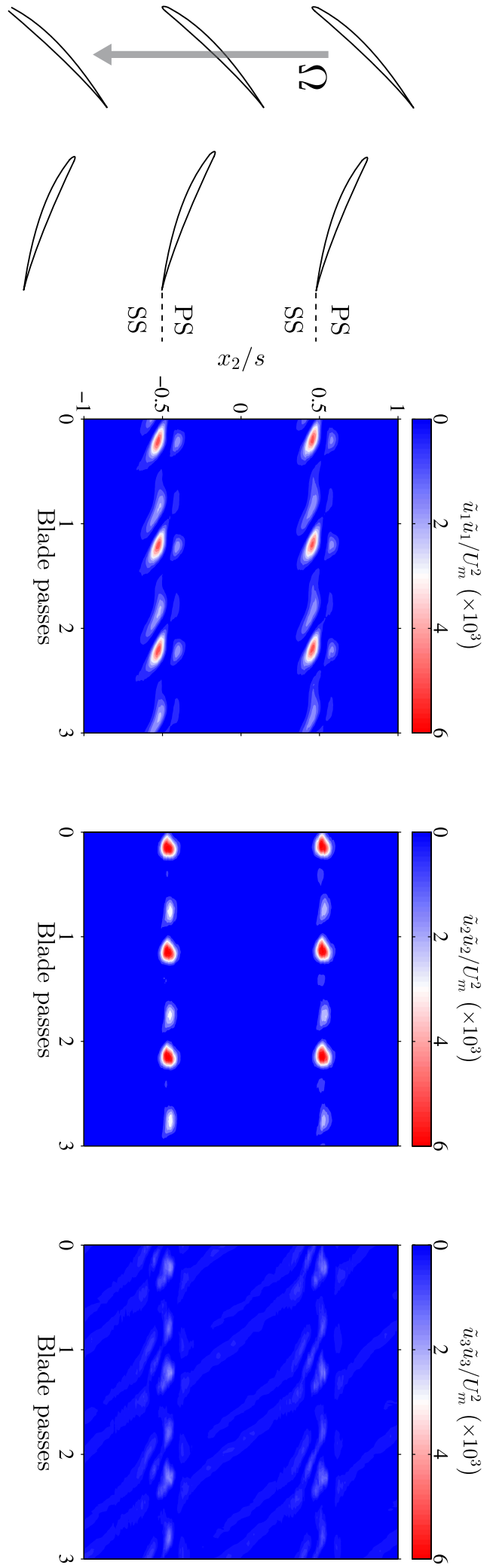

Fig. 16 Phase-averaged deterministic normal stresses including the axial-axial $\tilde{u}_{1} \tilde{u}_{1}$, tangential-tangential $\tilde{u}_{2} \tilde{u}_{2}$, and radial-radial $\tilde{u}_{3} \tilde{u}_{3}$ components. Note that data are shown for three blade passing periods and have been normalised using the rotor speed at mid-height, $U_{m}$, and the stator pitch, $s$ 

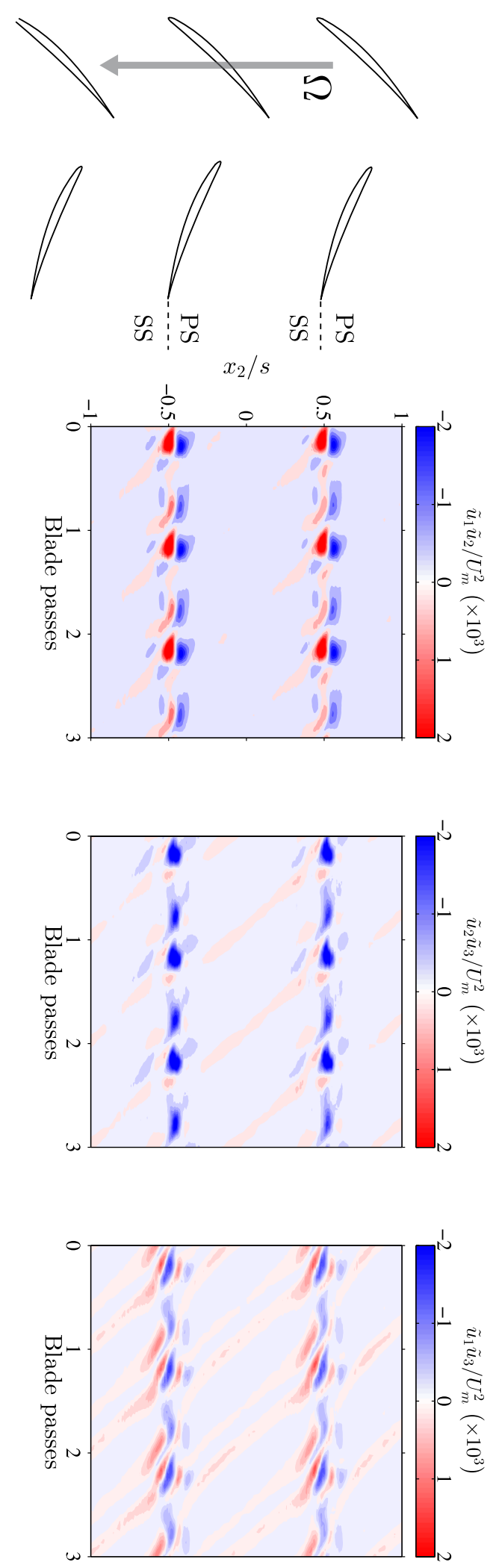

Fig. 17 Phase-averaged deterministic shear stresses including the axial-tangential $\tilde{u}_{1} \tilde{u}_{2}$; tangential-radial $\tilde{u}_{2} \tilde{u}_{3}$, and axial-radial $\tilde{u}_{1} \tilde{u}_{3}$ components. Note that data are shown for three blade passing periods and have been normalised using the rotor speed at mid-height, $U_{m}$, and the stator pitch, $s$ of normal stress statistics in the near-wake region (Hah and Lakshminarayana 1980). Finally, in the free-stream region, appreciable levels of Reynolds normal stress occur, which are in-phase with the passing rotor wakes. This observation implies that the deterministic flow structures support high levels of mean-squared stochastic unsteadiness in between adjacent stator wakes.

The maximum and minimum values of the turbulence intensity

$I^{\prime}=\frac{1}{U_{m}} \sqrt{\frac{1}{3} \overline{\left\langle u_{i}^{\prime} u_{i}^{\prime}\right\rangle}} \times 100$

were computed to be 6.5 and $2.5 \%$ and occur within the stator wake and free-stream regions, respectively. The circumferential average of turbulence intensity was computed to be $3.7 \%$, compared to $1.5 \%$ for the deterministic turbulence intensity.

The phase-averaged Reynolds shear stresses downstream of the compressor stator is shown in Fig. 19. Space-time contours of the axial-tangential, $\left\langle u_{1}^{\prime} u_{2}^{\prime}\right\rangle$, tangential-radial, $\left\langle u_{2}^{\prime} u_{3}^{\prime}\right\rangle$, and axial-radial, $\left\langle u_{1}^{\prime} u_{3}^{\prime}\right\rangle$, components are plotted for three blade passing periods. Relative to the deterministic shear stresses shown previously in Fig. 17, the Reynolds shear stresses show a significant reduction of intermittency and exhibit low-frequency meandering motion about the stator wake centre line. In terms of magnitude, the dominant Reynolds shear stress is the tangential-radial correlation, $\left\langle u_{2}^{\prime} u_{3}^{\prime}\right\rangle$, followed by the axial-radial correlation, $\left\langle u_{1}^{\prime} u_{3}^{\prime}\right\rangle$, and finally, the axial-tangential component, $\left\langle u_{1}^{\prime} u_{2}^{\prime}\right\rangle$

\section{Conclusions}

A rotated hot-wire method has been developed to characterise the unsteady, three-dimensional and multi-scale flow patterns which develop between compressor blade rows. Relative to past rotated hot-wire techniques, the current method benefits from superior levels of statistical fidelity and provides full details of the phase-averaged velocities, deterministic velocities, deterministic stresses, and Reynolds stresses. As a result, this method can be used to delineate the phase-averaged effect of rotor-stator interaction and non-linear turbulent convection. To improve the current understanding of momentum and energy exchange mechanisms in compressor annuli, the deterministic unsteadiness associated with the relative motion of blade rows must be separated from the genuine turbulence. This can be seen by considering the time-average of the phase-averaged Navier-Stokes equation: 

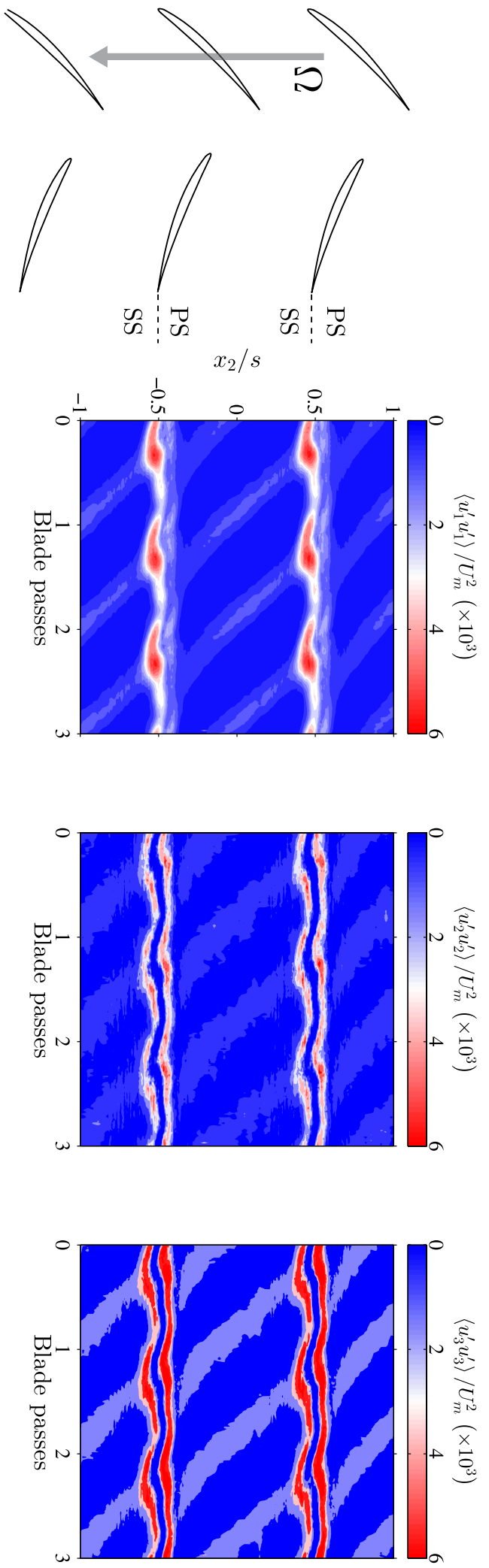

Fig. 18 Phase-averaged Reynolds normal stresses including axialaxial $\left\langle u_{1}^{\prime} u_{1}^{\prime}\right\rangle$, tangential-tangential $\left\langle u_{2}^{\prime} u_{2}^{\prime}\right\rangle$, and radial-radial $\left\langle u_{3}^{\prime} u_{3}^{\prime}\right\rangle$ components. Note that data are shown for three blade passing periods and have been normalised using the rotor speed at mid-height, $U_{m}$, and the stator pitch, $s$
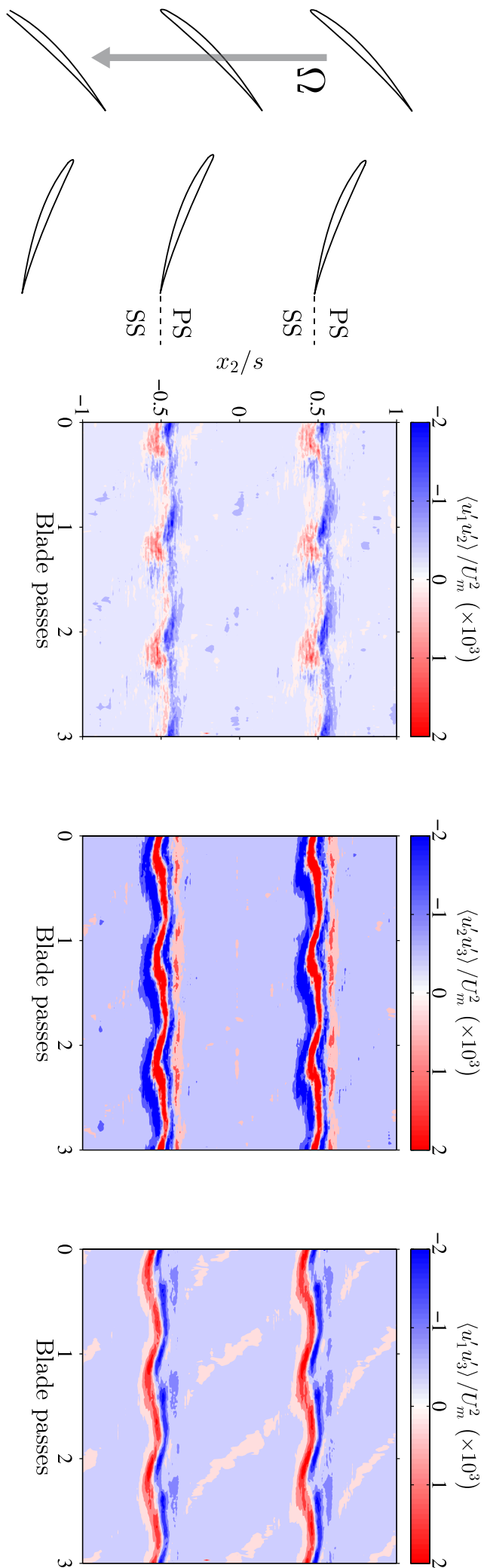

Fig. 19 Phase-averaged Reynolds shear stresses including axialtangential $\left\langle u_{1}^{\prime} u_{2}^{\prime}\right\rangle$, tangential-radial $\left\langle u_{2}^{\prime} u_{3}^{\prime}\right\rangle$, and axial-radial $\left\langle u_{1}^{\prime} u_{3}^{\prime}\right\rangle$ components. Note that data are shown for three blade passing periods and have been normalised using the rotor speed at mid-height, $U_{m}$, and the stator pitch, $s$ 
$\bar{u}_{j} \frac{\partial \bar{u}_{i}}{\partial x_{j}}=-\frac{\partial \bar{p}}{\partial x_{i}}+\frac{1}{R e} \frac{\partial^{2} \bar{u}_{i}}{\partial x_{j} \partial x_{j}}-\underbrace{\frac{\partial\left(\overline{\tilde{u}_{i} \tilde{u}_{j}}\right)}{\partial x_{j}}}_{I}-\underbrace{\frac{\partial\left(\overline{u_{i}^{\prime} u_{j}^{\prime}}\right)}{\partial x_{j}}}_{I I}$

where terms $I$ and $I I$ show the phase-integrated effect of the deterministic and Reynolds stresses against the mean flow, respectively. The competing effects of deterministic and stochastic unsteadiness upon compressor performance have been a hotly debated topic ever since the works of Adkins and Smith (1982) and Gallimore and Cumpsty (1986). Furthermore, the phase-locked statistics reported in this paper will enable comparisons against other scale-resolving measurement techniques, such as three-component particle image velocimetry (Bhattacharya et al. 2016), and high fidelity numerical simulations (Brandvik and Pullan 2009) to be made.

Full details of the theoretical, numerical, and experimental aspects of a new rotated hot-wire method are provided in this paper and include: (1) a complete derivation of the phase-averaged sensor response equations following a triple decomposition of the instantaneous field variables; (2) the numerical solution of the sensor response equations using a weighted non-linear regression algorithm and (3) a demonstration of the technique in a low-speed, single-stage compressor research facility. Rotated hot-wire measurements were acquired at mid-span, 20\% chord downstream from the stator trailing edge, whilst operating the compressor close to its design point. The phase-locked flow statistics reported in this paper reveal the space-time evolution of rotor-stator interactions (Figs. 14, 15), deterministic stresses (Figs. 16, 17), and Reynolds stresses (Figs. 18, 19) in the wake of a compressor stator. At mid-span, the relative magnitude of phase-integrated deterministic and Reynolds stress is comparable, whereas the relative magnitude of the phase-resolved fields can vary significantly. This variation is a due to the intermittency of the phase-averaged deterministic stresses-which become negligible for a large portion of each blade passing period. Unlike their deterministic counterpart, the Reynolds stresses exhibit a weaker phase dependence and remain more fully-developed within the stator wake.

Future measurement campaigns will focus on acquiring rotated hot-wire measurements in the compressor end-wall regions and downstream of the rotor. The relative magnitude of the deterministic and stochastic stress fields across the compressor operating map will also be studied. These data will be used to evaluate the appropriate terms in phase-averaged Reynolds stress transport equations which, ultimately, will lead to an improved understanding of transport phenomena in compressor flows.
Acknowledgements The authors gratefully acknowledge RollsRoyce plc and the UK TSB TUFT programme for funding this work and granting permission for its publication. All authors wish to thank Dr. J. J. Adamczyk, Dr. J. Bolger, Prof. N. A. Cumpsty, Dr. S. J. Gallimore, and Dr. J. S. S. Wong for their insightful comments and interest in this work. T.O.J. extends personal thanks to Mr. D. Barlow, Mrs. M. Folk, and Dr. H. C.-H. Ng for their assistance in the laboratory.

Open Access This article is distributed under the terms of the Creative Commons Attribution 4.0 International License (http:// creativecommons.org/licenses/by/4.0/), which permits unrestricted use, distribution, and reproduction in any medium, provided you give appropriate credit to the original author(s) and the source, provide a link to the Creative Commons license, and indicate if changes were made.

\section{References}

Adamczyk JJ (1985) Model equation for simulating flows in multistage turbomachinery. ASME Paper No. 85-GT-226

Adkins GG, Smith LH (1982) Spanwise mixing in axial flow turbomachines. J Eng Gas Turbines Power 104:97-110

Berdanier RA, Key NL (2016) A novel data reduction technique for single slanted hot-wire measurements used to study incompressible compressor tip leakage flows. Exp Fluids 57:29. doi:10.1007/ s00346-016-2114-z

Bhattacharya S, Berdanier RA, Vlachos PP, Key NL (2016) A new particle image velocimetry technique for turbomachinery applications. J Turbomach 138(12):124501

Bissonnette LR, Mellor GL (1974) Experiments on the behaviour of an axisymmetric turbulent boundary layer with a sudden circumferential strain. J Fluid Mech 63:369-413

Bradshaw P (1996) Turbulence modeling with application to turbomachinery. Prog Aerosp Sci 32:575-624

Bradshaw P, Cebeci T, Whitelaw JH (1981) Engineering calculation methods for turbulent flow. McGraw-Hill, London

Brandvik T, Pullan G (2009) An accelerated 3D Navier-Stokes solver for flows in turbomachines. ASME Turbo Expo 2009; Power for Land, Sea, and Air; GT2009-60052

Brundrett E, Baines WD (1964) The production and diffusion of vorticity in duct flow. J Fluid Mech 19:375-394

Buresti G, Di Cocco NR (1987) Hot-wire measurement procedures and their appraisal through a simulation technique. J Phys E Sci Instrum 20:87-99

Dawes WN (1991) Multi-blade row Navier-Stokes simulation of fanbypass configurations. ASME paper 91-GT-148

De Grande G, Kool P (1981) An improved experimental method to determine the complete Reynolds stress tensor with a single rotating hot wire. J Phys E Sci Instrum 14:196-201

Denton JD (1990) The calculation of three dimensional viscous flow through multisage turbomachines. ASME paper 92-GT-364

Denton JD (2010) Some limitations of turbomachinery CFD. ASME Turbo Expo 2010; Power for Land, Sea, and Air; GT2010-22540

Díaz KA, Oro JF, Vega MG, Marigorta EB (2016) Effects of prongwire interferences in dual hot-wire probes on the measurements of unsteady flows and turbulence in low-speed axial fans. Measurement 91:1-11

Dryden HL (1938) Turbulence investigations at the National Bureau of Standards. Appl Mech Int 5th Congr 5:362-368

Faure TM, Thierry M, Michon G, Miton H, Vassilieff N (2001) Laser Doppler anemometry measurements in an axial compressor stage. J Propuls Power 17(3):481-491 
Fujita H, Kovasznay LSG (1968) Measurement of Reynolds stress by a single rotated hot wire anemometer. Phys Fluids 39:1351-1355

Gallimore SJ, Cumpsty NA (1986) Spanwise mixing in multi-stage axial flow compressors Part I. Experimental investigation. J Turbomach 108:2-9

Georgiou DP, Milidonis KF (2014) Fabrication and calibration of a sub-miniature 5-hole probe with embedded pressure sensors for use in extremely confined and complex flow areas in turbomachinery research facilities. Flow Meas Instrum 39:54-63

Goto A (1991) Three-dimensional flow and mixing in an axial flow compressor with different rotor tip clearances. ASME 1991 international gas turbine and aeroengine congress and exposition; 91-GT-89 1

Grimshaw S, Taylor J (2016) Fast settling millimetre-scale five-hole probes. In: ASME Turbo Expo 2016: Turbomachinery technical conference and exposition, American Society of Mechanical Engineers, pp V006T05A014-V006T05A014

Hah C, Lakshminarayana B (1980) Measurment and prediction of mean velocity and turbulence structure in the near wake of an airfoil. J Fluid Mech 115:251-282

Hinze JO (1959) Turbulence. McGraw-Hill, New York

Hsu S, Wo A (1997) Near-wake measurement in a rotor/stator axial compressor using slanted hot-wire technique. Exp Fluids 23(5):441-444

Hussain AKMF, Reynolds WC (1970) The mechanics of an organized wave in turbulent shear flow. J Fluid Mech 41(02):241-258

Inoue M, Kuromaru M (1984) Three-dimensional structure and decay of vortices behind an axial flow rotating blade row. ASME J Eng Gas Turbines Power 106:561-569

Jørgensen FE (1971) Directional sensitivity of wire and fibre-film probes. DISA information No. 11

King L (1914) Small cylinders in a stream of fluid: determination of the convection constants of small platinum wires with applications to hot-wire anemometry. Phil Trans R Soc (Lond) 214:373-432

Kuroumaru M, Inoue M, Higaki T, Abd-Elkhalek F, Ikui T (1982) Measurement of three dimensional flow field behind an impeller by means of periodic multi-sampling with a slanted hot wire. Bull JSME 39:1674-1681

Lakshminarayana B (1981) Techniques for aerodynamic and turbulence measurements in turbomachinery rotor. J Eng Power 103:374-392

Lakshminarayana B, Poncet A (1974) A method of measuring threedimensional rotating wakes behind turbomachinery rotors. ASME J Fluids Eng 96:87-91
Ma H, Jiang H (2004) An experimental study of three-dimensional characteristics of turbulent wakes of axial compressor rotors. J Therm Sci 14:15-21

di Mare L, Jelly TO, Day IJ (2017) Angular response of hot wire probes. Meas Sci Technol 28(3):035303

Papavegros PG, Hedley AB (1979) A simple practical method for establishing turbulence characteristics by means of a single 45 degrees slant hot-wire probe in a field of known mean flow direction. J Phys E Sci Instrum 12:761-765

Prandtl L (1946) On boundary layers in three-dimensional flow. Reports and Transactions, No. 64, British M.A.P

Rai MM (1989) Three-dimensional Navier-Stokes simulations of turbine rotor-stator interaction. part i-methodology. J Propuls Power 5(3):305-311

Reynolds WC, Hussain AKMF (1972) The mechanics of an organized wave in turbulent shear flow. Part 3 . Theoretical models and comparisons with experiments. J Fluid Mech 54:263-288

Schmidt DP, Okiishi TH (1977) Multistage axial flow turbomachinery wake production, transport and interaction. AIAA J 15:1138-1145

Shin H, Whitfield CE, Wisler DC (1994) Rotor-rotor interaction for counter-rotating fans, Part 1: Three-dimensional flowfield measurements. AIAA J 32:2224-2233

Sieverding HC, Arts T, Dénos R, Brouckaert JF (2000) Measurement techniques for unsteady flows in turbomachines. Exp Fluids 28(4):285-321

Sirivat A (1989) Measurement and interpretation of space-time correlation functions and derivative statistics from a rotating hot wire in grid turbulence. Exp Fluids 7:361-370

Treaster LA, Yocum AM (1978) The calibration and application of five-hole probes. DTIC Document

Uzol O, Chow Y, Meneveau C (2002) Experimental investigation of unsteady flow field within a two-stage axial turbomachine using particle image velocimetry. ASME J Turbomach 124:542-552

Webster CAG (1962) A note on the sensitivity to yaw of a hot-wire anemometer. J Fluid Mech 20:307-312

Weichert S, Day I (2014) Detailed measurements of spike formation in an axial compressor. J Turbomach 136(5):051006

Zierke WC, Okiishi TH (1982) Measurement and analysis of totalpressure unsteadiness data from an axial-flow compressor stage. J Eng Power 104(2):479-488 